\title{
Macrophages use a bet-hedging strategy for antimicrobial activity in phagolysosomal acidification
}

\author{
Quigly Dragotakes, ${ }^{1}$ Kaitlin M. Stouffer, ${ }^{1}$ Man Shun Fu, ${ }^{1}$ Yehonatan Sella, ${ }^{2}$ Christine Youn, ${ }^{3}$ Olivia Insun Yoon, ${ }^{4}$ \\ Carlos M. De Leon-Rodriguez, ${ }^{1}$ Joudeh B. Freij, ${ }^{1}$ Aviv Bergman, ${ }^{2,5}$ and Arturo Casadevall' \\ 'Department of Molecular Microbiology and Immunology, Johns Hopkins School of Public Health, Baltimore, Maryland, USA. ²Department of Systems and Computational Biology, Albert Einstein College \\ of Medicine, Bronx, New York, USA. ${ }^{3}$ Department of Dermatology, Johns Hopkins School of Medicine, Baltimore, Maryland, USA. ${ }^{4}$ Johns Hopkins University, Krieger School of Arts and Sciences, Baltimore, \\ Maryland, USA. ${ }^{5}$ Santa Fe Institute, Santa Fe, New Mexico, USA.
}

\begin{abstract}
Microbial ingestion by a macrophage results in the formation of an acidic phagolysosome but the host cell has no information on the $\mathrm{pH}$ susceptibility of the ingested organism. This poses a problem for the macrophage and raises the fundamental question of how the phagocytic cell optimizes the acidification process to prevail. We analyzed the dynamical distribution of phagolysosomal pH in murine and human macrophages that had ingested live or dead Cryptococcus neoformans cells, or inert beads. Phagolysosomal acidification produced a range of $\mathrm{pH}$ values that approximated normal distributions, but these differed from normality depending on ingested particle type. Analysis of the increments of $\mathrm{pH}$ reduction revealed no forbidden ordinal patterns, implying that the phagosomal acidification process was a stochastic dynamical system. Using simulation modeling, we determined that by stochastically acidifying a phagolysosome to a $\mathrm{pH}$ within the observed distribution, macrophages sacrificed a small amount of overall fitness to gain the benefit of reduced variation in fitness. Hence, chance in the final phagosomal pH introduces unpredictability to the outcome of the macrophage-microbe, which implies a bet-hedging strategy that benefits the macrophage. While bet hedging is common in biological systems at the organism level, our results show its use at the organelle and cellular level.
\end{abstract}

\section{Introduction}

Audaces fortuna iuvat (fortune favors the bold)

$$
\text { - Virgil }
$$

Phagocytosis is a fundamental cellular process used by unicellular organisms for nutrient acquisition as well as by host immune cells for microbial defense. The parallels between food acquisition and immunity have led to the suggestion that these 2 processes had a common evolutionary origin (1). The process of phagocytosis results in the formation of a phagolysosome, a dynamic membrane-bounded organelle, which represents a critical arena in the struggle between the host and ingested microbial cells (2). Microbial ingestion into phagosomes results in exposure to host cell microbicidal mechanisms, which leads to death for some microbes. Others survive by subverting critical aspects of phagosome maturation and by damaging phagolysosome structural integrity.

The process of phagosomal maturation, encompassed by the fusion of the phagosome with lysosomes and lumen acidification, is a complex choreography that includes the recruitment of V-ATPase from lysosomes to the phagolysosome $(2,3)$ and many other protein components (4). Proton pumping into the phagolysosomal lumen results in acidification that inhibits microbes and activates

Authorship note: $A B$ and $A C$ share senior authorship.

Conflict of interest: The authors have declared that no conflict of interest exists. Copyright: @ 2020, American Society for Clinical Investigation.

Submitted: September 30, 2019; Accepted: April 10, 2020; Published: June 8, 2020.

Reference information: / Clin Invest. 2020;130(7):3805-3819.

https://doi.org/10.1172/JCl133938. antimicrobial processes. Consequently, some types of microbes, such as Mycobacterium tuberculosis and Histoplasma capsulatum, interfere with phagosomal maturation and acidification to promote their intracellular survival. The extent of phagosomal acidification is determined by numerous mechanisms that include proton flux through the pump, proton consumption in the phagosomal lumen, and backflow into the cytoplasm (5). Phagosome acidification in macrophages is rapid, with a $\mathrm{pH}$ of 6 being reached within 10 minutes after ingestion (6) and a $\mathrm{pH}$ of 5.4 being reached by 15 to 20 minutes (7).

Cryptococcus neoformans is a facultative intracellular pathogen (8). Upon ingestion by macrophages, C. neoformans resides in a mature acidic phagolysosome (9). The outcome of macrophage-C. neoformans interaction is highly variable depending on whether the fungal cell is killed, inhibited, or unaltered. If not killed, C. neoformans can replicate intracellularly, resulting in death and lysis of the host cell, nonlytic exocytosis $(10,11)$, transfer to another macrophage $(12,13)$, or phagosomal persistence. Maintenance of phagolysosomal membrane integrity is a critical variable in determining the outcome of the C. neoformans-macrophage interaction, with loss of integrity leading to host cell death (14).

Prior studies of C. neoformans phagosomal acidification measured great variation in the $\mathrm{pH}$ of individual phagolysosomes (1416). The $\mathrm{pH}$ of cryptococcal phagolysosomes is affected by several microbial factors, including urease expression (15), phagosomal membrane integrity (14), and the presence of the cryptococcal capsule with glucuronic acid residues (17). In addition, the capsule 
A
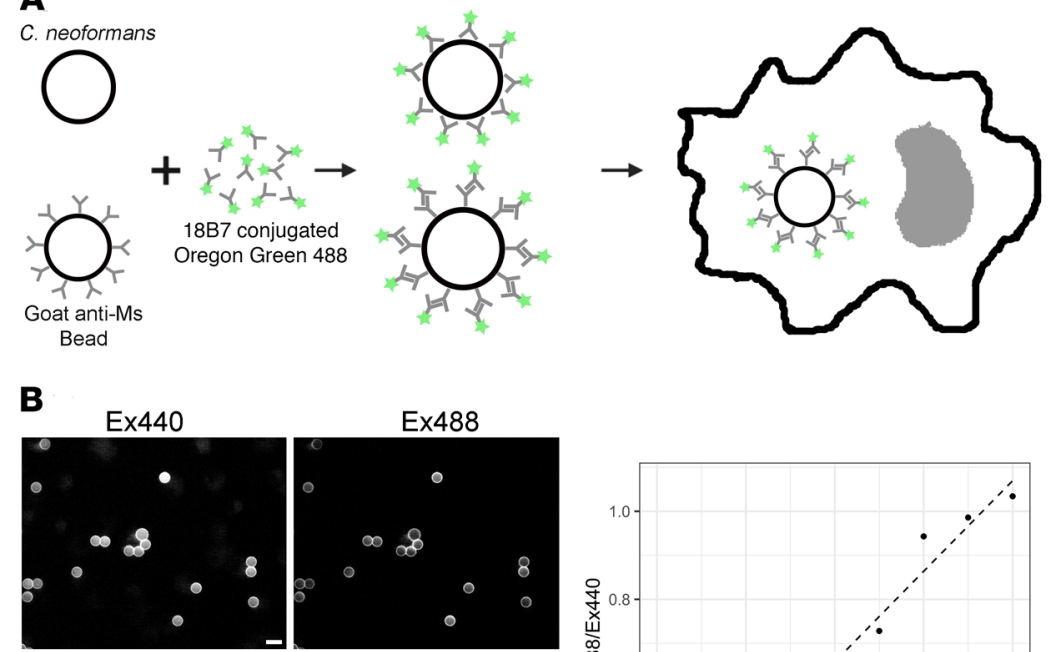

Ratio
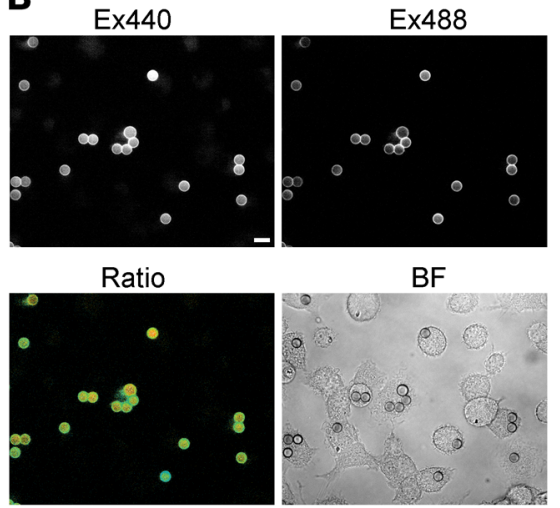

$\mathrm{BF}$
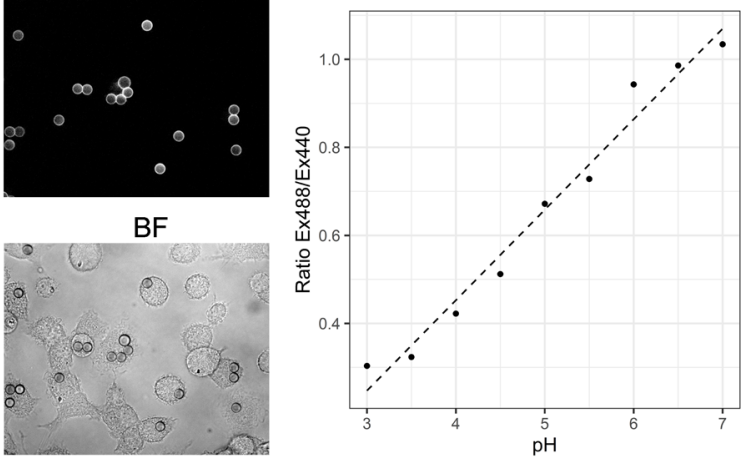

C Scanning window

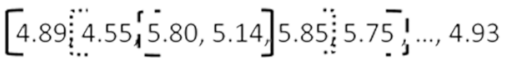

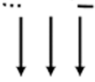

Window values Ordinal pattern

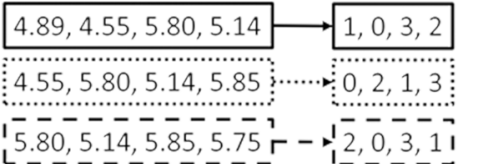

Figure 1. Experimental designs for described methods. (A) Experimental design for phagolysosomal pH measurement. Particles are opsonized with mAb $18 \mathrm{~B} 7$ conjugated to fluorophore Oregon Green 488. (B) Measurement of phagolysosomal pH. Measurements are based on ratiometric measuring of Ex440/Em520 and Ex488/Em520. Scale bar: $10 \mu \mathrm{m}$. (C) Experimental design for the detection of chaos within a system. A scanning window generates sets of windows values. Ordinal patterns are then generated by ordering the terms in each scanning window from least to greatest.

of C. neoformans increases in diameter as part of a stress response that can potentially affect the phagolysosomal $\mathrm{pH}$ through increasing the phagolysosome volume, thus diluting its contents and promoting membrane damage through physical stress (14).

In this study, we analyzed the time dependence of the phagolysosomal $\mathrm{pH}$ distribution in murine and human macrophages and concluded that acidification is a stochastic process in which macrophages default to a range of $\mathrm{pHs}$ with random variation. We demonstrate that, in doing so, macrophages employ a bet-hedging strategy by sacrificing a small amount of overall fitness to ensure broad survivability. Thus, we suggest that chance, in the form of stochastic dynamics, could be an important strategy that benefits the macrophage by bet hedging against a range of pathogens, and could echo through the immune process to introduce a fundamental uncertainty in the outcome of microbemacrophage interactions.

\section{Results}

We previously reported a wide distribution of phagolysosomal $\mathrm{pH}$ after the ingestion of C. neoformans by murine bone marrow-derived macrophages (BMDMs) $(14,15)$. Given that the growth rate of C. neoformans is highly affected by $\mathrm{pH}(15,18)$ and that the outcome of the C. neoformans-macrophage interaction is determined in the phagolysosome (14, 19, 20), we decided to analyze the distribution of phagolysosomal $\mathrm{pH}$ mathematically to gain insight into the dynamics of the acidification process. Understanding the fundamental process of phagolysosomal acidification, and if there are overarching rules or determinism involved, could provide key insights to disease pathology of any pathogen, which would interact with the phagolysosome. A scheme of the method used to determine phagosomal acidification with representative data from polystyrene bead phagocytosis experiments is shown in Figure 1A. To determine the $\mathrm{pH}$ of a phagolysosome, particles are opsonized with an Oregon Green-conjugated Ab. Oregon Green is $\mathrm{pH}$ insensitive at Ex440/Em520 and $\mathrm{pH}$ sensitive at Ex488/Em520, allowing $\mathrm{pH}$ to be measured by quantifying the ratio of fluorescence intensity between each wavelength. We were able to establish robust standard curves from known $\mathrm{pH}$ controls and calculate unknown phagolysosome measurements (Figure 1B).

Murine macrophage phagolysosomes acidify stochastically. To determine whether phagolysosomal acidification is a deterministic or stochastic dynamical process, we employed a permutation spectrum test (21) in which the distribution of ordinal patterns occurring in subsets of our full data set was analyzed (Figure 1C). Ordinal patterns simply refer to the order of each measurement in terms of value, in our case the phagolysosomal $\mathrm{pH}$, within a scanning window, which parses all measurements within each condition, exemplified in Figure 1 (22-25). Here we found a 4 -unit window size to be the most appropriate. We found no forbidden patterns at any time evaluated for any of the $\mathrm{pH}$ distributions resulting from the synchronized ingestion of beads, alive C. neoformans, or heat-killed C. neoformans, which implies that the acidification is a stochastic process (Figure 2). A forbidden ordinal is an ordinal pattern that does not appear during the time frame of our experiment. Despite the overall population of macrophages showing chaotic dynamics, we considered whether the chaotic signatures occurred at the individual cellular level. That is, whether slight differences at the initiation of phagocytosis propagated through maturation and acidification resulting in 2 phagolysosomes within the same cell with different acidifications. To determine if this was the case, we analyzed the phagosomal $\mathrm{pH}$ in pairs of bead-containing phagolysosomes for which each pair was within a single macrophage. We chose macrophages in which the ingested beads were visibly separated. We found that the differences between phagolysosomal $\mathrm{pH}$ measurements of this cohort of phagolysosomes yields a normal distribu- 


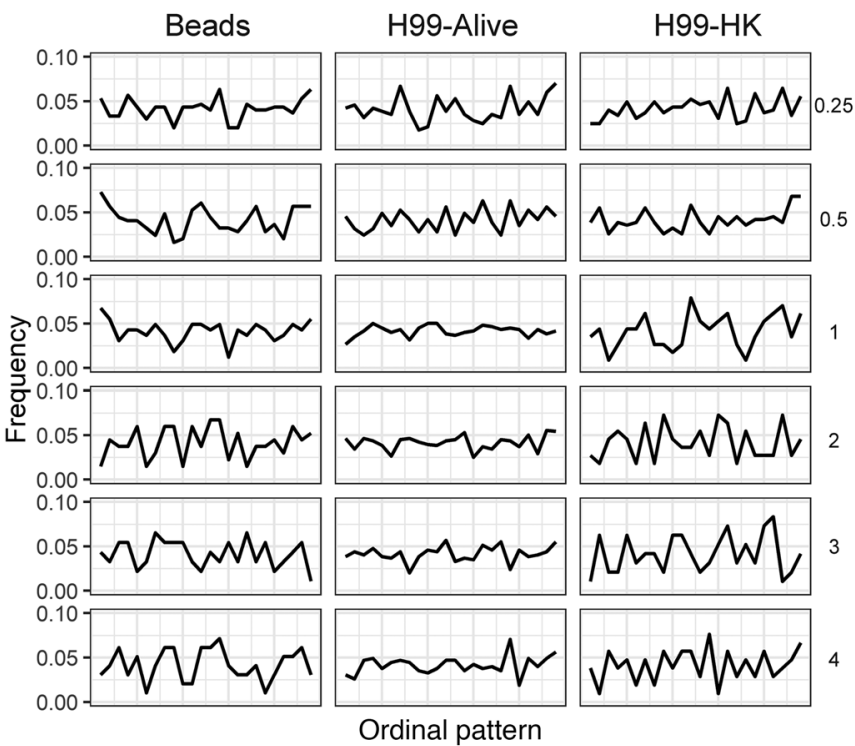

Figure 2. Murine macrophage phagolysosomes acidify stochastically. The frequency of each 4-unit ordinal pattern for each ingested particle: beads, live $C$. neoformans, and dead $C$. neoformans at various HPI. Note that all ordinal patterns have a non-zero frequency.

tion centered at 0 with non-zero values, suggesting each individual phagosome has an independent target phagolysosomal pH (Supplemental Figure 1; supplemental material available online with this article; https://doi.org/10.1172/JCI133938DS1). The ordinal pattern analysis was repeated on several other strains and conditions throughout the project and at no point did we observe forbidden ordinal patterns (Supplemental Figure 2A), again suggesting the process exhibits no signature of deterministic chaos (22-25). Given that beads are inert and cannot modify $\mathrm{pH}$, we reasoned that their corresponding phagolysosomes would be the closest approximation of a default acidification state and would therefore represent a baseline to which we could compare phagolysosome acidification dynamics in other conditions.

Bead-ingested murine macrophage phagolysosomes stabilize to a normally distributed $\mathrm{pH}$. To probe the dynamics of the phagolysosomal acidification system at this baseline, we analyzed several hundred individual phagolysosomal $\mathrm{pH}$ measurements at various time intervals after BMDMs had ingested inert beads (Figure 3A). To determine whether phagolysosome $\mathrm{pH}$ measurements followed a normal distribution, the measured relative $\mathrm{pH}$ values were fit to a predicted normal distribution using the fitdistrplus R statistical package then analyzed via quantile-quantile $(\mathrm{Q}-\mathrm{Q})$ plots and the Shapiro-Wilk normality test. We found that phagolysosome $\mathrm{pH}$ value distributions did not approximate normality at early times (15 and 30 minutes), but mostly did at intervals of and past 1 hour after infection. We were able to reject the Shapiro-Wilk null hypothesis only at 15 minutes, 30 minutes, and 3 hours (Figure 3B). We hypothesized that not all phagolysosomes were fully mature before 1 hour and reasoned that the bimodal appearance of $\mathrm{pH}$ at early time intervals is likely due to the population of phagosomes being at different stages of maturity. Interestingly, the $\mathrm{pH}$ distribution for the phagolysosomes containing live or dead C. neoformans did not approximate normality even at later times, nor did other live yeast-containing phagolysosomes (Supplemental Figure 3). We rejected Shapiro-Wilk normality for each of these samples except for dead C. neoformans at 3 hours. Q-Q plots supported the notion that each sample skews further from normality compared with bead-ingested macrophage phagolysosome $\mathrm{pH}$ distributions (Supplemental Figure 4). Considering that macrophages default to a random $\mathrm{pH}$ from a particular normal distribution and that $C$. neoformans invest in disrupting this process, we hypothesized that this system must confer some host benefit. Thus, we decided to compare the $\mathrm{pH}$ distribution of mature bead-ingested phagolysosomes to a range of $\mathrm{pHs}$ tolerated by relevant potential pathogens.

Acidification dynamics are closely related to maturation. To probe whether the observed normality and stochasticity is a result of dynamics in acidification or in the maturation process, we analyzed the intensity of 2 phagolysosomal maturation markers, EEA1 and V-ATPase, over the same time course. Using relative intensity of each immunofluorescent staining as a surrogate measurement of maturation, we found that, again, no samples had forbidden ordinal patterns, suggesting phagolysosomal maturation, when measured by the accumulation of these markers, is also stochastic in nature (Supplemental Figure 5). However, neither of the fluorescence intensity measurements from these markers approximated normality at any time, with sufficient skewing to reject the Shapiro-Wilk null hypothesis (Supplemental Figure 6). Skewing of these measurements away from normality could reflect cytoplasmic speckling, limitations of fluorescence microscopy resolution, nonlinearity of fluorescence signals, and the inherent complexity of such a system, such that we are not confident to reject the notion that these processes indeed demonstrate a normal distribution. We hypothesized that macrophages may experience limited resources in terms of the number of available V-ATPase pumps at any given time and that we might see a correlation between intensity of VATP staining around individual phagosomes in cells that had ingested one versus multiple beads. We found no evidence of a correlation between V-ATPase staining, or EEA1 for that matter, and total number of ingested particles (Supplemental Figure 7).

$B M D M$ phagolysosomes acidify to a $\mathrm{pH}$ range suboptimal for growth of soil and pathogenic microbes. The $\mathrm{pH}$ of soil varies greatly from acidic to alkaline based on a variety of conditions that, in turn, determine the associated microbiome (26). Soil contains many pathogenic microbes including C. neoformans. Since the phagolysosome is an acidic environment, we reasoned that microbes that thrive in acidic soil could proxy for the types of microbes that hosts, and thus macrophages, could encounter, and pose a threat to the cell/host due to their acidophilic nature (e.g., C. neoformans). Hence, we compared $(27,28)$ the distribution of $\mathrm{pH}$ values from mature phagolysosomes (HPI $\geq 1$ hour) that had ingested latex beads, as a measure of the range of acidities generated in the absence of microbial modulation, relative to published soil microbe growth data as a function of $\mathrm{pH}$ (Figure 4). The latex bead $\mathrm{pH}$ distribution is narrow and centered at a $\mathrm{pH}$ of about 4.5 , which corresponds to a $\mathrm{pH}$ that significantly reduces the optimal growth even for microbes in acidic soils. To generate a more relevant comparison to human disease models, we also searched out the $\mathrm{pH}$ tolerance of 27 substantial human pathogens that macrophages are likely to encounter during human infectious diseases (Supplemental Table 1). We then compared these distributions to the $\mathrm{pH}$ distribution in bead- 
A
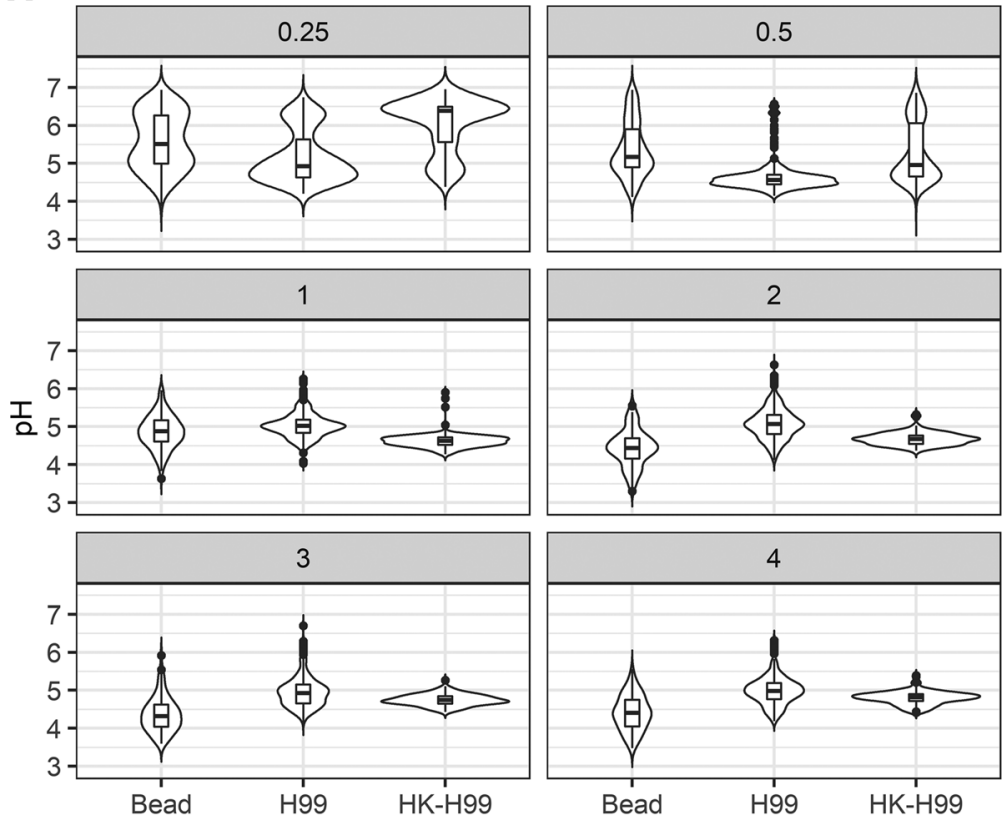

Figure 3. Bead-ingested murine macrophage phagolysosomes stabilize to a normally distributed pH. (A) Distributions of measured phagolysosomal $\mathrm{pH}$ after ingestion of various particles by BMDMs at various HPI (gray box value) for bead, live C. neoformans (H99), and dead C. neoformans (HK-H99). (B) Visualization of estimated normality via Shapiro-Wilk (line) and total phagolysosome count (bar) for each sample.

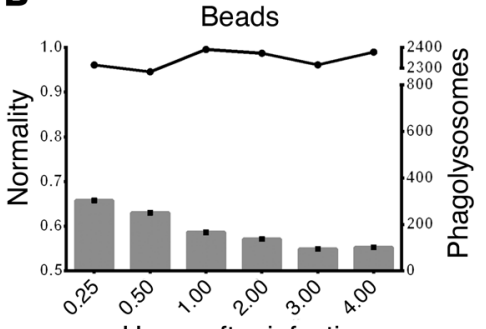

Hours after infection

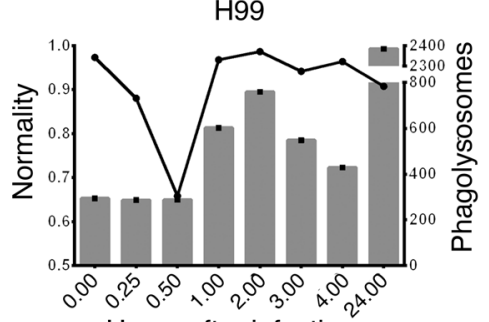

Hours after infection

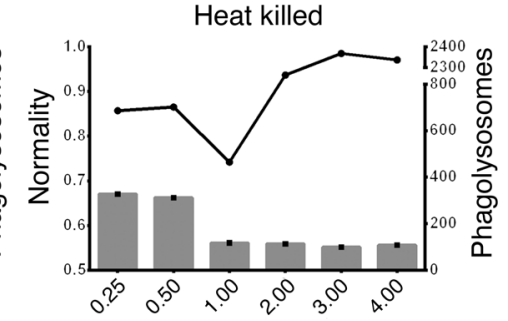

Hours after infection ingested macrophage phagolysosomes (Figure 4). The distribution of this default macrophage $\mathrm{pH}$ heavily overlaps with the more inhibitory $\mathrm{pH}$ regions when compared with both soil and known pathogen $\mathrm{pH}$ tolerances. Taken together, these data suggest that the low phagosomal $\mathrm{pH}$ is itself a defense mechanism, with a distribution that manifests bet hedging by defaulting to a range, rather than to a single value, inhibitory to most of the spectrum of pathogens the macrophage is likely to encounter.

Simulations of macrophage populations show stochastic $\mathrm{pH}$ as a bet-hedging strategy. Bet hedging is generally understood as a strategy whereby an organism decreases variation in fitness at the expense of a small decrease in mean fitness. To test whether variation in macrophage phagolysosome $\mathrm{pH}$ constitutes a diversified bet-hedging strategy, we first modeled host survival rate as a function of final phagolysosomal $\mathrm{pH}$ in the context of a pathogen randomly selected from Supplemental Table 1. This analysis modeled phagolysosomal $\mathrm{pH}$ as a normal distribution with mean $\mu$ and standard deviation $\sigma$ varied over a range of possible values in order to test how bet hedging depends on these parameters. To each phagolysosomal $\mathrm{pH}$, we associated a fitness value that models survival likelihood against pathogens, using a list of pathogens (Supplemental Table 1) with their viable $\mathrm{pH}$ ranges collected from the literature, and we computed the distribution of fitness (p) as phagolysosomal $\mathrm{pH}$ varies (Figure $5, \mathrm{~A}$ and $\mathrm{B}$ ). As expected in bet hedging, we observed decreases in standard deviation of fitness with increasing $\sigma$ of phagolysosomal $\mathrm{pH}$ (Figure $5 \mathrm{C}$ ). Mean fitness was mostly unchanged with changing standard deviation, such that even in the most extreme deviations in phagolysosomal $\mathrm{pH}$ there were only slight changes in fitness in either direction.

Another way to formalize the emergence of bet hedging as an evolutionary strategy is by considering the average long-term rate of growth. Thus, we considered a multiplicative model, in which the growth rate $\left(r_{T}\right)$ over a span of time $(\mathrm{T})$ is defined as the geometric mean of $\rho_{1}$ through $\rho_{T}$, where for each $\mathrm{t}$ ranging from 1 to $\mathrm{T}, \rho_{\mathrm{t}}$ is the fitness at time t. Taking $\operatorname{logs}, \log \left(r_{T}\right)$ is the average of $\log \left(\rho_{1}\right)$ through $\log \left(\rho_{t}\right)$. By the law of large numbers, if we assume each $\rho_{t}$ is independent and identically distributed, this average will approach $\mathrm{E}(\log (\rho))$. Thus, $\mathrm{E}(\log (\rho))$ is the operative quantity to be maximized, rather than $E(\rho)$. Note that applying the log transformation has the effect of placing heavier penalties on fitness values that are close to 0 , so that maximizing mean log fitness will tend to encourage lower standard deviation in fitness (29). Thus, increased mean log fitness is another indication of bet hedging that directly relates to long-term growth. We indeed observe increased mean log fitness with increasing $\sigma$ of phagolysosomal pH (Figure 5D).

To probe whether our simulations would reflect biological responses, we analyzed macrophage phagolysosomal $\mathrm{pH}$ in which cells were treated with chloroquine, a weak base that localizes to the phagolysosome. According to our model, the increased 


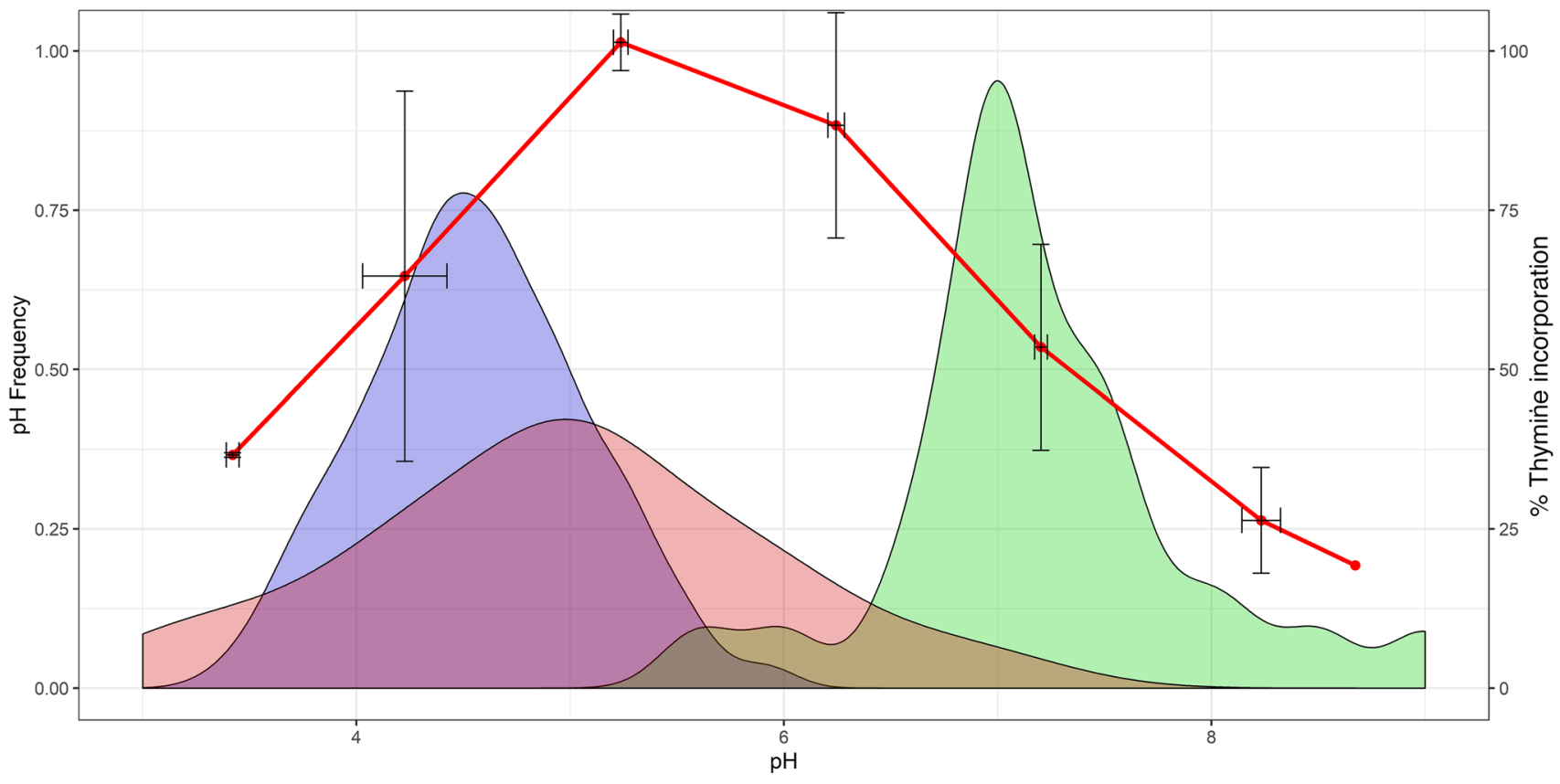

Figure 4. BMDM phagolysosomes acidify to a default pH range suboptimal for soil microbe and pathogen growth. Growth rates of soil bacteria (27, 28) as percentage of thymine incorporation (red line) and distribution of minimal culturable pH for 27 human pathogens (red fill) compared with the distribution of observed phagolysosomal pH after bead ingestion and phagolysosome maturation, 1 to $4 \mathrm{HPI}$ (blue fill). The bead pH distribution overlaps unfavorably with minimal growth conditions of microbes, and only minimally overlaps with the optimal growth pH range of the same 27 pathogens (green fill).

shift in mean $\mathrm{pH}$ from chloroquine would result in a lower overall mean $\log$ fitness as a result of shifting the mean $\mathrm{pH}$ closer to 6 , a more tolerable region for most of the candidate pathogens. Thus, we compared previously reported data (9) of C. neoformanscontaining phagolysosomes treated with chloroquine to our data from the respective time interval and found that chloroquine treatment results in a drastically reduced overall mean log fitness $(-5.323)$ compared with the mean log fitness of our non-chloroquine-treated data of the respective condition (-2.486).

Time intervals from ingestion of C. neoformans to initial budding are stochastic. The $C$. neoformans replication rate is highly dependent on $\mathrm{pH}$ (15). Consequently, we hypothesized that if phagolysosomal acidification followed stochastic dynamics, this would be reflected on the time interval from ingestion to initial replication. Analysis of time intervals to initial fungal cell budding events revealed stochastic dynamics with no evidence of forbidden ordinal patterns (Supplemental Figure 2B). Similar results were observed for initial budding of WT and urease negative strains of C. neoformans, which reside in phagolysosomes that differ in final $\mathrm{pH}$ as a result of ammonia generation from urea hydrolysis. Acidification intervals for both strains were stochastic, despite the fact that phagolysosomes of urease-deficient strains are approximately $0.5 \mathrm{pH}$ units lower than those of WT strains (15).

Trained murine macrophages have inverse acidification dynamics. Trained immunity has recently been shown to influence repeated infection in monocyte populations not exposed to the adaptive immune system (30). To determine whether initial exposure to a pathogen has an effect in the dynamics of this system, we exposed BMDMs to C. neoformans, resolved the infection with antifungals, and measured phagolysosome acidification dynamics upon reinfection. We found that $\mathrm{pH}$ distribution of phagolysosomes from macrophages previously trained as described also exhibited stochastic behavior (Figure 6A), veering away from a normal distribution of pH (Figure 6B and Supplemental Figure 8). However, trained BMDMs on average exhibited a significantly lower initial $\mathrm{pH}$, which became significantly higher compared with untrained BMDMs over time (Figure 6C). Fully understanding this system will require significant further study outside the scope of this manuscript. In this regard, we note that amphotericin B is a powerful activator of macrophages (31) and that $C$. neoformans residence inside macrophages is associated with host cell damage $(32,33)$. Hence, the effects we observe could be the aggregate of several influences in the system. Nevertheless, there is a clear suggestion of a historical effect on which $\mathrm{pH}$ distribution a macrophage will employ. This may also suggest an adaptive component to the macrophage bet-hedging strategy.

Differently polarized macrophages acidify stochastically but not using this bet-hedging system. To probe whether M0 and M2 polarized macrophages acidify with the same dynamics of M1 macrophages, we repeated these experiments with macrophages that were either not stimulated, or stimulated with IL-4 to skew toward M2. First, we found that regardless of the polarization skew, all macrophages acidified stochastically (Figure 7A). Second, we found that the phagolysosomal $\mathrm{pH}$ distributions differed overall, with M2 macrophages having the highest mean $\mathrm{pH}$, followed by $\mathrm{MO}$ - and then by M1-skewed macrophages (Figure 7B). Most striking was the observation that MO- and M2-skewed macrophages did not manifest a normally distributed $\mathrm{pH}$ range as observed with M1-skewed macrophages. Instead, MO macrophages consistently yield a bimodal distribution even after 1 hour. The phagosomal $\mathrm{pH}$ distribution of M2 macrophages started with a heavy tail of higher $\mathrm{pH}$ and eventually stabilized to a bimodal distribution (Supplemental Figure 9). These 
A

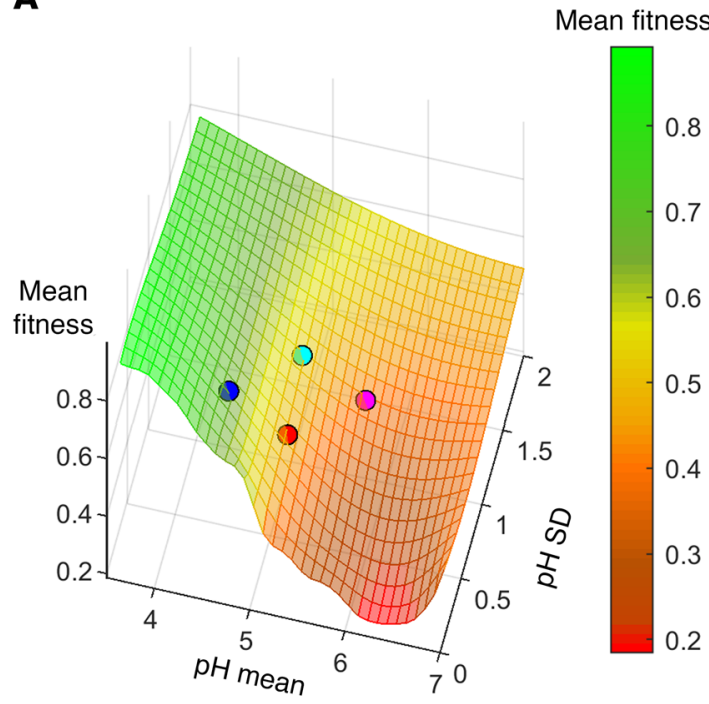

C

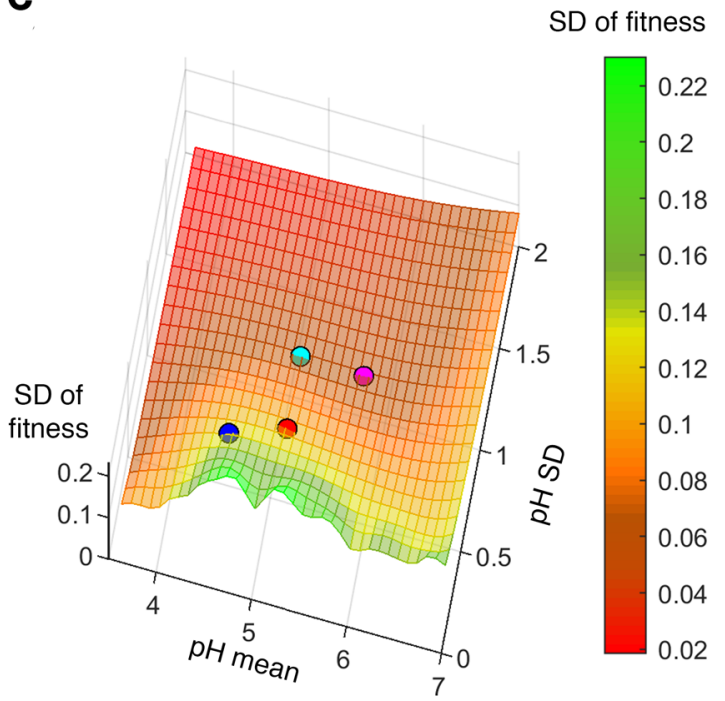

B

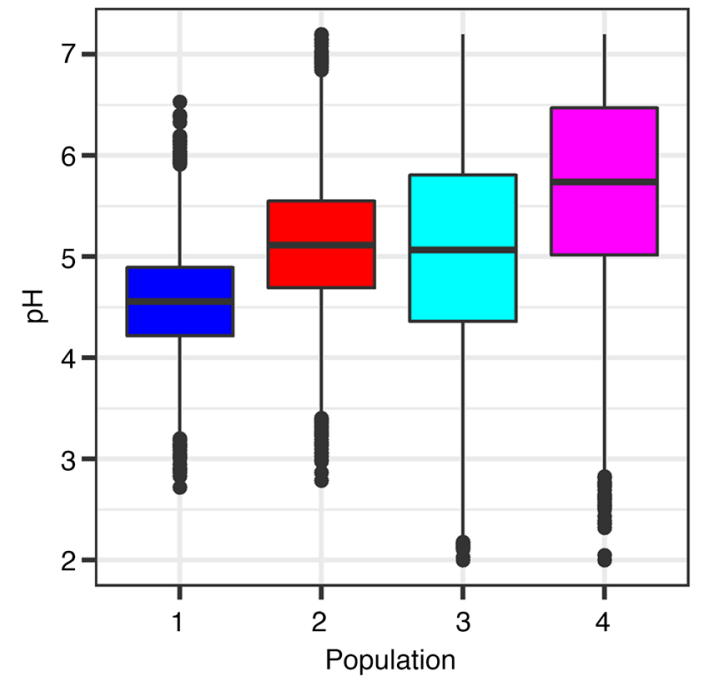

D

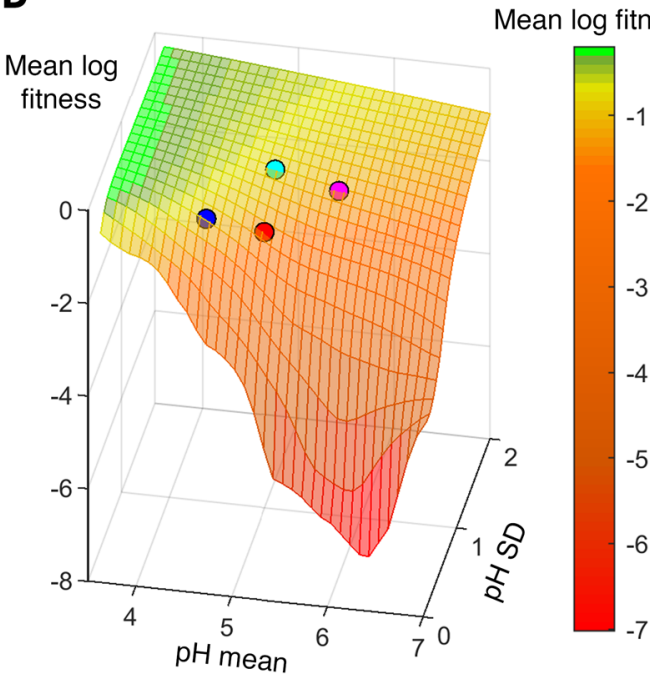

Figure 5. Simulated macrophage populations show stochastic pH as bet-hedging strategy. Success of host-microbe interactions are visualized as macrophage populations faced with randomly selected pathogens and acidified to random pHs from normal distributions of mean $\mu$ and standard deviation $\sigma$. Meshes represent host macrophage fitness, deviation in fitness, and log mean fitness. Plotted points represent measured data of H99-containing murine (red) and human (magenta) or bead-containing murine (blue) and human (cyan) phagolysosomes. (A) Mean macrophage survival ( $z$ axis, color bar) increases significantly as pH lowers, and mostly unchanged with standard deviation. (B) Examples of simulated populations based on colored points in $\mathbf{A}$. Each combination of mean and SD from the axes of $\mathbf{A}$ represent a unique population of macrophages with a fitness represented by the $Z$ axis and colored mesh. (C) Deviation in host fitness ( $z$ axis, color bar) dramatically decreases by increasing standard deviation of $\mathrm{pH}$, and mostly unaffected by shifts in mean pH. (D) Logarithmic measurement of host fitness ( $z$ axis, color bar) to observe long term trends applicable to a bet-hedging strategy.

data suggest that while the macrophages have the same underlying acidification dynamics, they do not share the betting strategy of M1-skewed macrophages. Thus, we estimated the likelihood of each population of macrophages to survive when faced with the same list of human pathogens modeled after bimodal distributions estimated from the observed data (Supplemental Figure 10). After comparing these simulations, we found that $\mathrm{M} 1$ macrophages by far have the highest mean log fitness, followed by M0, then by M2 (Figure 7C). Our model shows M1 macrophages as resistant to these infectious agents, whereas M2 macrophages are permissive.

Human monocytes acidify stochastically and approximate normality. To determine how closely the murine system resembled human acidification dynamics, we isolated macrophages from human peripheral blood monocytes and repeated these experiments with beads and live C. neoformans. We found that acidification intervals in human cells were also stochastic in nature (Figure 8A). Additionally, human cells that ingested inert beads were normally distributed at the 15-minute and 1-hour time intervals. Even though the times skewed away from normality, the skew was not as severe as that observed in yeast-containing phagolysosomes (Figure $8, \mathrm{~B}$ and $\mathrm{C}$ ). We hypothesize that some of this skewing could result from different dynamics due to the different background inherent to human donors, which differ from the mouse system in which cells are isolated from genetically identical individuals (Supplemental Figure 11). Furthermore, within the context of our simulation, if we model the phagolysosome $\mathrm{pH}$ values of a population of 


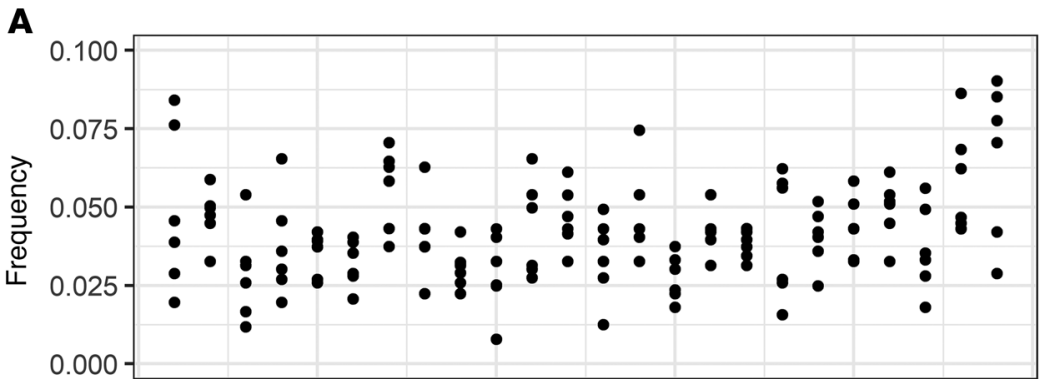

Ordinal pattern

B

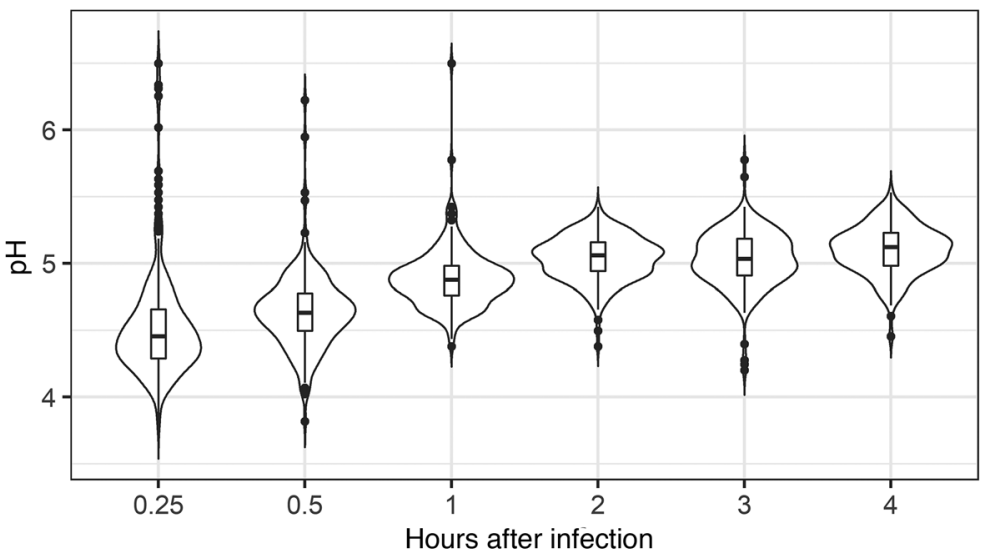

C

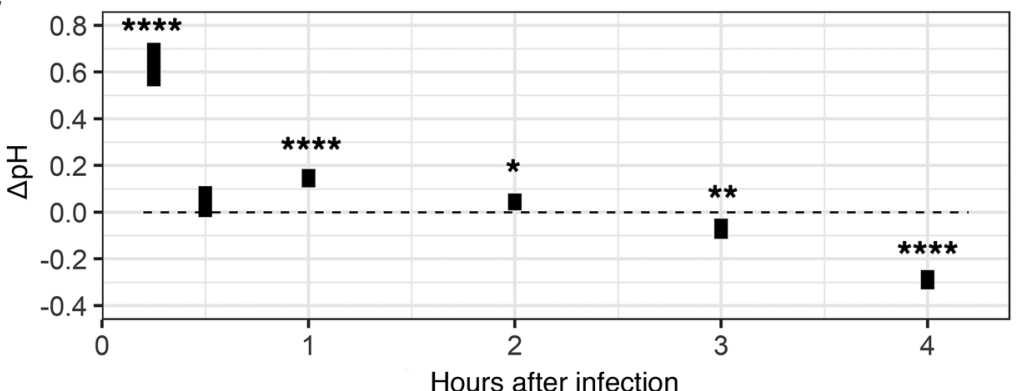

Figure 6. Trained murine macrophages have inverse acidification dynamics. (A) Ordinal pattern analysis of phagolysosome $\mathrm{pH}$ in trained BMDMs. All ordinal patterns appear at a non-zero frequency. (B) pH distributions of phagolysosomes at various HPI. (C) Difference $(95 \% \mathrm{Cl})$ after subtracting mean trained phagolysosome $\mathrm{pH}$ from mean untrained phagolysosome $\mathrm{pH}$ at each time point. ${ }^{*} P<0.05$; ${ }^{* *} P<0.01$; ${ }^{* * *} P<0.0001$ via 2 -tailed $t$ test.

lower, which inhibits C. neoformans replication (34), and calculated the likelihood of each population of macrophages to achieve an inhibitory $\mathrm{pH}$, assuming phagolysosome pHs were normally distributed around the observed mean and standard deviation. Here we analyzed the combined data for all time intervals, reasoning that in actual infection interactions between $C$. neoformans and host macrophages early phagosomes would be important as well, rather than focusing only on mature phagolysosomes.

We estimated the proportions of inhibitory phagolysosomes and found that bead-containing phagolysosomes were more likely to acidify to 4 or lower, followed by heat-killed and $\Delta$ ure1 C. neoformans, followed by live C. neoformans, with capsuledeficient Cap59 being particularly likely, and trained macrophages or C. gattii-containing phagolysosomes being particularly unlikely to achieve inhibitory $\mathrm{pH}$ (Figure 9A). Our actual data were not normally distributed with non-bead samples though, as C. neoformans actively modulates phagolysosomal $\mathrm{pH}$. The starkest difference we observed is that, in reality, killed and live C. neoformans phagolysosomes had the same proportion of inhibitory phagolysosomes, suggesting the capsule has a more substantial effect on $\mathrm{pH}$ modulation than we initially expected. This finding is corroborated by the high proportion of inhibitory phagolysosomes in Cap59-containing phagolysosomes, a strain incapable of modulating $\mathrm{pH}$ since it has no capsule (Figure 9C). Additionally, the expected and observed increased likelihood of bead-containing phagolysosomes to inhibit $C$. neoformans replication compared with live $C$. neoformans phagolysosomes was consistent between murine and human cells (Figure 9, B and D).

To probe whether this phenomenon was applicable to pathogens other than C. neoformans, we repeated this analysis with published phagolysosomal $\mathrm{pH}$ data from Mycobacterium avium (35). We found that like C. neoformans, live M. avium bacteria modified their resident phagolysosomal $\mathrm{pH}$ to be more favorable toward them. In contrast, the estimated mean log fitness for the host macrophage

human macrophages as a normal distribution with mean and standard deviation determined from $\mathrm{pH}$ values observed across all time points (5.08 and 1.08, respectively), the resulting mean log fitness is high with dramatically reduced deviation in fitness (Figure 5).

Pathogens skew phagolysosome acidification toward conditions less favorable to the macrophage. Cryptococcal cells buffer the phagolysosome $\mathrm{pH}$ toward 5.5, a value optimal for yeast growth (14). We therefore hypothesized that C. neoformans could use this buffering capability to disrupt the host acidification strategy. We found that phagolysosomes containing live C. neoformans and live C. gattii acidified to distributions of $\mathrm{pH}$ less normally distributed and more permissive to general fungal replication by increasing the average observed phagolysosomal $\mathrm{pH}$.

To probe host fitness with regard to C. neoformans specifically, we modified our model by declaring the inhibitory $\mathrm{pH}$ as 4 or population increased when they ingested killed mycobacteria, which are unable to modulate $\mathrm{pH}$ (Supplemental Figure 12).

\section{Discussion}

The complexity and sequential nature of the phagolysosomal maturation process combined with the potential for variability at each of the maturation steps, and the noisy nature of the signaling networks that regulate this process, have led to the proposal that each phagolysosome is a unique and individual unit (36). In fact, the action of kinesin and dynein motors that move phagosomes along microtubules exhibits stochastic behavior, adding another source of randomness to the process (37). Hence, even when the ingested particle is a latex bead taken through one specific type of phagocytic receptor, there is considerable heterogeneity in phagolysosome composition, even within a single cell (36). Since the phagolyso- 
A

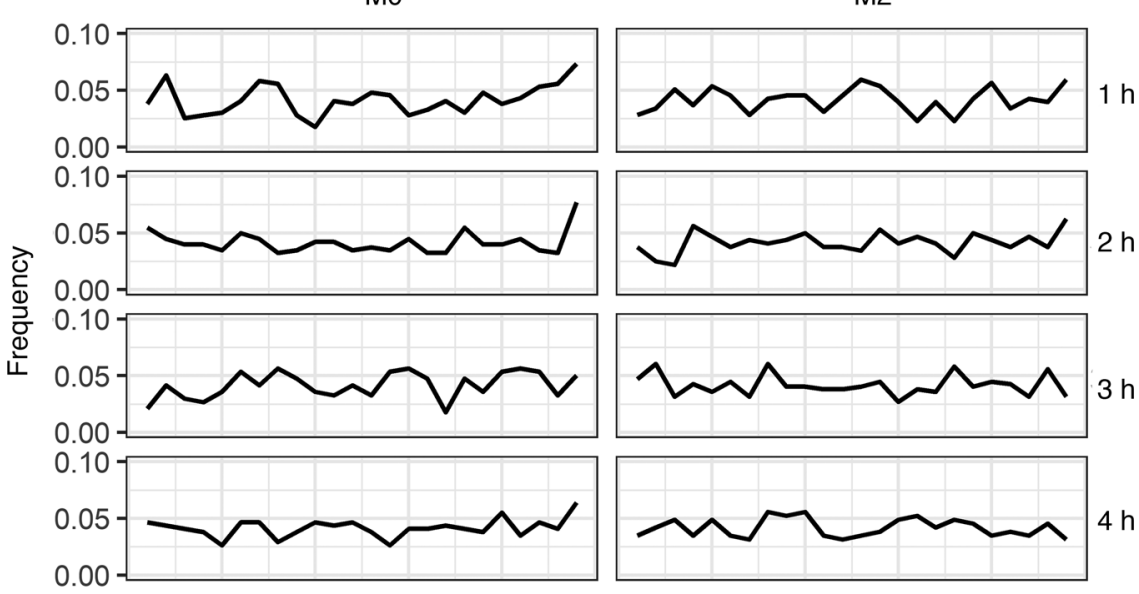

B

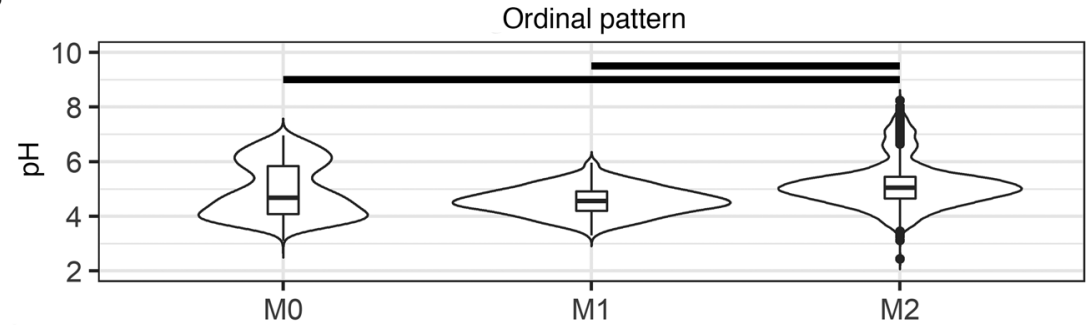

C

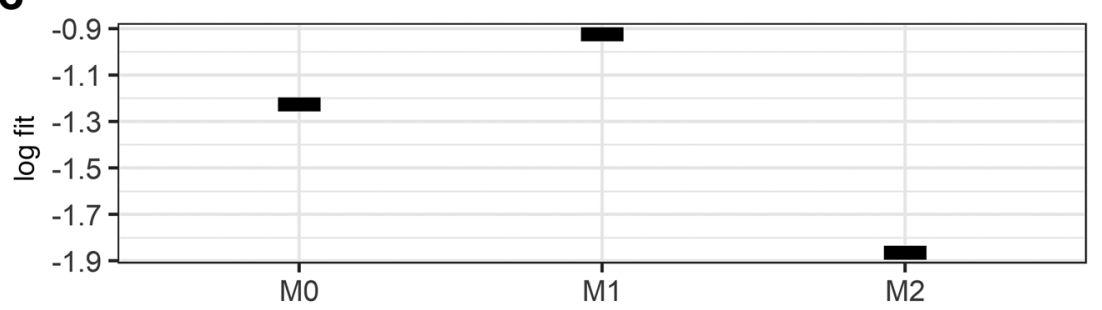

Figure 7. Phagolysosome dynamics of macrophages skewed toward different polarization states. (A) Ordinal patterns of bead-containing phagolysosomes at various HPI. (B) Bead-containing phagolysosome $\mathrm{pH}$ distributions of differently polarized macrophages. Each group represents measurements from all time points (1-4 HPI) of each polarization. Black bars represent $P<0.0001$ via Kruskal-Wallis test with Wilcox rank pairing test. (C) Mean log fitness of bead-containing phagolysosomes according to our bet-hedging model.

some is a killing machine used to control ingested microbes, this heterogeneity implies there will be differences in the microbicidal efficacy of individual phagolysosomes. This variability raises fundamental questions about the nature of the dynamical system embodied in the process of phagosomal maturation.

In this study, we analyzed the dynamics of phagolysosome $\mathrm{pH}$ variability after synchronized ingestion of live yeast cells, dead yeast cells, and latex particles. We sought to characterize the acidification dynamics as either stochastic, an inherently unpredictable process with identical starting conditions yielding different trajectories in time, or deterministic, a theoretically predictable process with identical starting conditions leading to identical trajectories. In particular, we focused our analysis on differentiating stochastic versus chaotic signatures in the trajectories of phagolysosomal $\mathrm{pH}$. While both dynamics might yield highly divergent trajectories for similar starting conditions (i.e., only 1 of 100 variables differ- ing by only a minuscule amount), a chaotic system is inherently deterministic, such that if strictly identical starting conditions were replicated, the same trajectory would follow from those conditions each time. A chaotic system is defined as one so sensitive to initial conditions that, in practice, initial conditions cannot be replicated precisely enough to see these same trajectories followed. The dynamical signatures of such systems are unique and can be differentiated from that of other deterministic or stochastic dynamics.

Irrespective of the nature of the ingested particle, we observed that the distribution of the increment of phagolysosomal $\mathrm{pH}$ reduction was random, indicative of a stochastic process. We found no evidence that phagosome acidification was a chaotic process. Systems in which a large number of variables each contribute to an outcome tend to exhibit noise, which gives them the characteristics of a stochastic dynamical system. Additionally, while particle size and shape affect ingestion time (38) the mean times to ingestion for beads and $C$. neoformans particles have been shown to be 2.5 and 4.18 minutes, respectively $(38,39)$. Since phagocytosis was synchronized and our observations were made over a period of hours, it is unlikely that the effects described here are due to noise from differences in uptake time differences. In this regard, our finding that phagolysosomal $\mathrm{pH}$ demonstrates stochastic features is consistent with our current understanding of the mechanisms involved. Q-Q plots revealed that most phagolysosomal $\mathrm{pH}$ distributions in this study manifested significant $(P<0.05$ via Shapiro-Wilk test) deviations from normality in several instances. Specifically, this stochastic normal distribution was generated at the phagolysosomal level, as evidenced by the fact that different phagosomes within the same macrophage manifested different $\mathrm{pH}$ values. Hence, each macrophage contains phagolysosomes with a different $\mathrm{pH}$ rather than each macrophage containing multiple of the same $\mathrm{pH}$, such that the normal distribution observed was generated at the organelle level. The most normally distributed $\mathrm{pH}$ sets were those resulting from the ingestion of latex beads, particles that cannot modify the acidity of the phagolysosome. We note that for the 3 C. gattii strains, the $\mathrm{pH}$ distributions revealed more skewing in Q-Q plots than for the H99 C. neoformans strain. Although the cause of this variation is not understood and the strain sample size is too small to draw firm conclusions, we note that such variation could reflect more microbial-mediated modification of the phagolysosomal $\mathrm{pH}$ by the $C$. gattii strains. In this regard, the capsular polysaccharide of the $C$. gattii strains has polysaccharide triads that are more complex (40) and, given that the cryptococcal polysaccharide capsule contains 
A

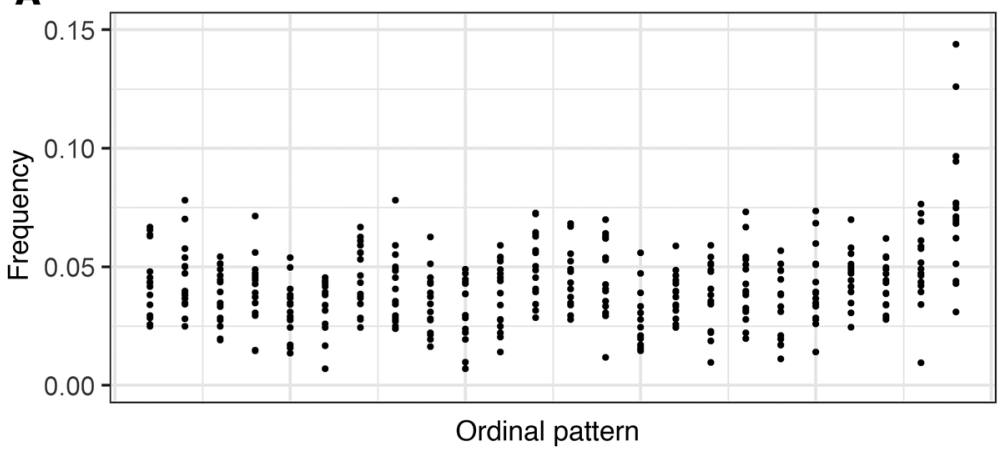

B
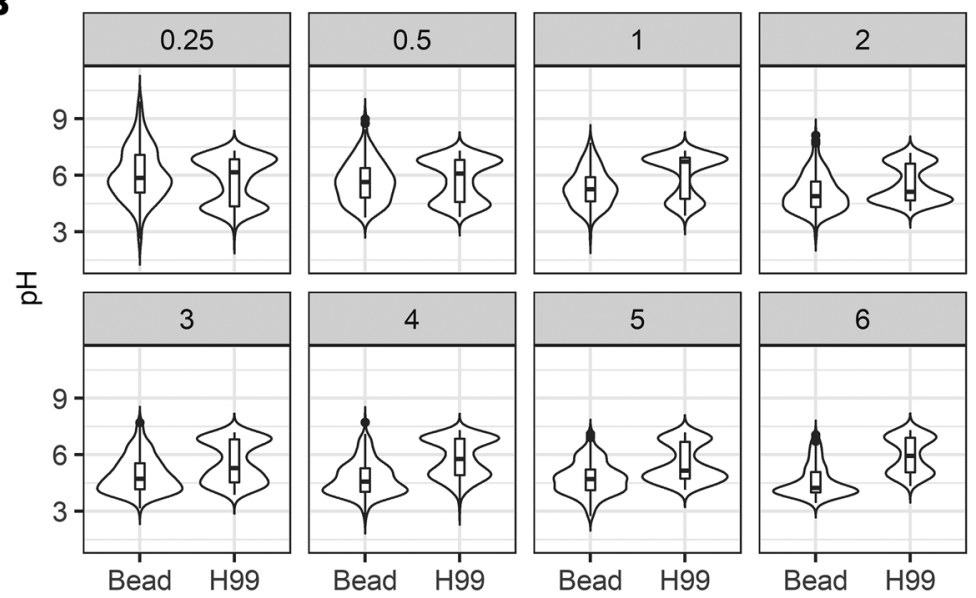

C

Bead

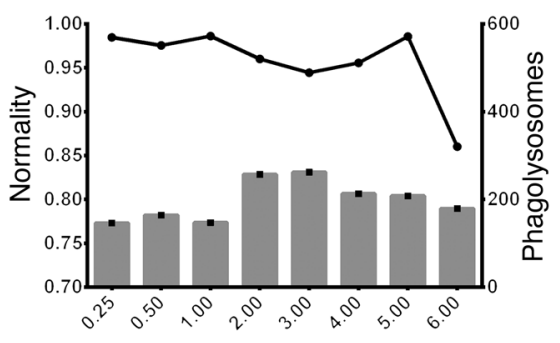

Hours after infection

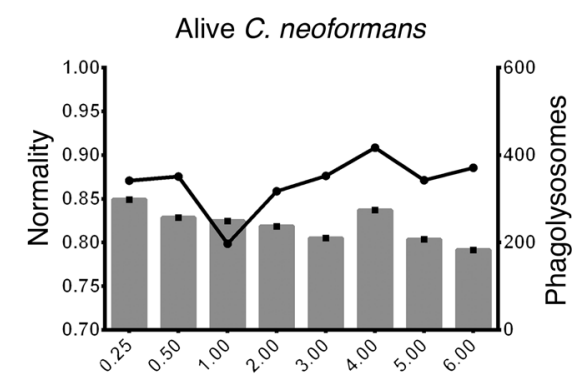

Hours after infection

Figure 8. Human monocyte-derived macrophages acidify stochastically and approximate normality. Human macrophages were infected with inert beads or live $C$. neoformans, and their $\mathrm{pH}$ analyzed at various time points. (A) Ordinal pattern analysis for all conditions. All patterns exist at non-zero frequencies for all time points. (B) Distributions of phagolysosome $\mathrm{pH}$ at different time points. (C) Shapiro-Wilk normality and total sample count for each condition.

is responsible for pumping protons into the phagolysosome and maintaining acidity. If the number of V-ATPase molecules translated directly to the number of protons pumped into the phagolysosome we would expect correlation between V-ATPase staining intensity and $\mathrm{pH}$, and thus expect normal distributions in both. The different dynamics observed with V-ATPase immunofluorescence implies that the $\mathrm{pH}$ heterogeneity is regulated by additional mechanisms. For example, it is possible that the efficacy of the V-ATPase pumps on the phagolysosomal membrane differs from pump to pump and that these differences also contribute to the distribution of phagolysosomal pHs observed.

For most microbes, maintenance of an acidic environment in the phagolysosome is critically determined on the integrity of the phagolysosomal membrane, keeping protons in the phagolysosomal lumen while excluding more alkaline cytoplasmic contents. For example, with C. albicans, the rupture of the phagolysosomal membrane is followed by rapid alkalization of the phagolysosomal lumen (41). For C. neoformans, phagolysosomal integrity is compromised by secretion of phospholipases that damage membranes as well as the physical stress on membranes resulting from capsular enlargement in the phagolysosome (14). However, for C. neoformans, loss of phagolysosomal membrane integrity does not immediately result in loss of phagolysosomal acidity, which is attributed to buffering by glucuronic acid residues in the $C$. neoformans capsule (17). Adding to the complexity of the C.neoformans-macrophage interaction is the fact that the phagolysosomal $\mathrm{pH}$ in the vicinity of 5.5 matches the optimal replication $\mathrm{pH}$ for this fungus (15), which can be expected to place additional stress on the organelle membrane through the increased volume resulting from budding cells. Treating macrophages with chloroquine, which

glucuronic acids that can modify phagolysosomal $\mathrm{pH}$ via acidbase properties (17), it is possible that this skewing reflects differences in phagolysosome-to-phagosome capsular effects.

We attempted to separate the dynamics of phagolysosomal maturation from acidification by investigating the accumulation of phagolysosomal maturation markers EEA1 and V-ATPase after ingestion. Analyzing the same time intervals where $\mathrm{pH}$ populations stabilized, namely 1 to 4 hours after ingestion, we found no forbidden ordinal patterns, suggesting that phagosome maturation was also a stochastic process. However, the acquisition of these 2 maturation markers did not approximate a normal distribution at any of the 4 time intervals, with all samples manifesting heavy right skewing. This is especially interesting considering V-ATPase increases phagosomal $\mathrm{pH}$ (42), potentiates macrophage antifungal activity against $C$. neoformans (43). Hence, phagosomal acidification does not inhibit $C$. neoformans replication but is critical for activation of mechanisms involved in antigen presentation (44). In the cryptococcal-containing phagolysosome the luminal $\mathrm{pH}$ is also likely to reflect a variety of microbial-mediated variables which include ammonia generation from urease, capsular composition, and the integrity of the phagolysosomal membrane.

Analysis of the normality of phagolysosomal $\mathrm{pH}$ distributions as a function of time by the Shapiro-Wilk test produced additional insights into the dynamics of these systems. Phagolysosomes containing inert beads manifested $\mathrm{pH}$ distributions that met criteria for normality at most time intervals after 1 hour post infection (HPI). 
A

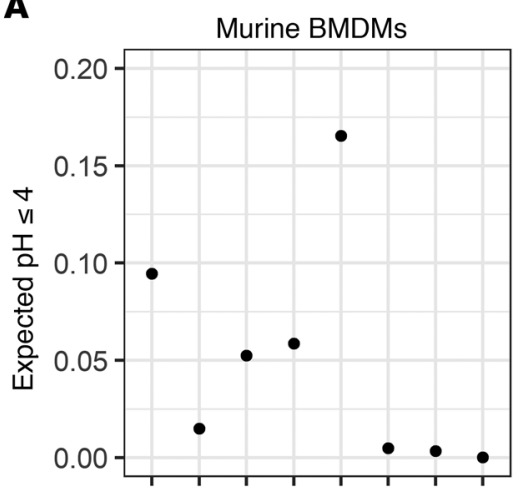

C

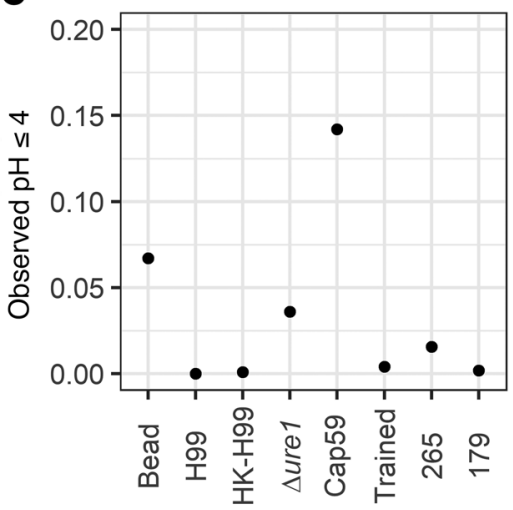

B

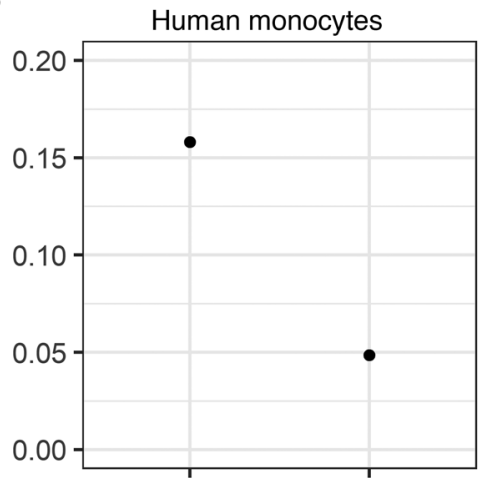

D

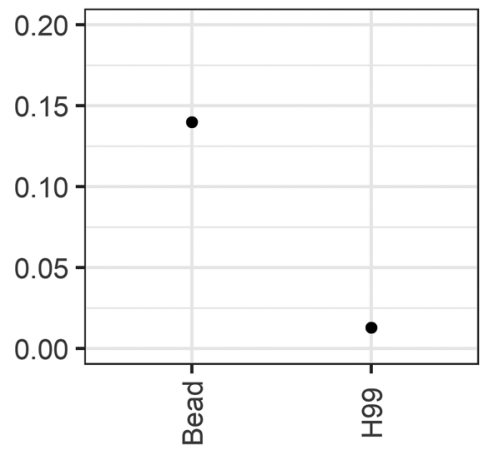

ride, which moved the phagolysosomal $\mathrm{pH}$ distribution toward normality. A similar effect may have occurred with strains R265, W179, and ure14. Convergence to or away from normality could reflect a myriad of such variables affecting phagolysosomal $\mathrm{pH}$, including the intensity of acidification, the volume of the phagolysosome (largely determined by the yeast capsule radius), the glucuronic acid composition of the capsule, the production of ammonia by urease, and the leakiness of the phagolysosome to cytoplasmic contents with higher $\mathrm{pH}$. Although our experiments cannot sort out the individual contributions of these factors, they suggest that, in combination, they produce Gaussian noise effects that push or pull the resulting distribution to or from normality. Additionally, human phagolysosome acidification dynamics resembled those of mouse cells but noted significant differences in the distributions of phagolysosomal $\mathrm{pH}$ between individual human donors. This donor-to-donor variation could reflect differences in polymorphisms in Fc receptor genes or other genetic variables and is an interesting subject for future studies.

When a phagocytic cell ingests a microbe, it has no information as to the $\mathrm{pH}$ range tolerated by the internalized microbe. A stochastic dynamical processforphagolysosomalacidification could provide phagocytic immune cells and their hosts with the best chance for controlling ingested microbes. The acidic $\mathrm{pH}$ in the phagolysosome activates microbicidal mechanisms and acidity is not generally considered a major antimicrobial mechanism in itself. However, our analysis of $\mathrm{pH}$ tolerances of 27 pathogenic microbes revealed that the majority are inhibited by phagolysosomal $\mathrm{pH}$ with the caveat that some, like C. neoformans (18) and Salmonella typhimurium (48), thrive in acidified

We hypothesize that at 0.25 and $0.5 \mathrm{HPI}$ there are 2 populations of phagolysosomes, mature and maturing, with the latter having not yet fully acidified and thus resulting in the observed bimodal distributions. Additionally, it has been shown that phagolysosomes of macrophages undergo active alkalization, regulated in part by NOX2 activity (45-47). It is likely that these early time points veer away from normality due to a combination of phagolysosomes maturing at a different rate and a subpopulation of phagolysosomes that are actively alkalized, both contributing noise to early phagolysosomal dynamics. In contrast, the $\mathrm{pH}$ distribution of phagolysosomes containing dead C. neoformans cells initially veered away from normality at 15 minutes, 30 minutes, and 1 hour but in later time intervals approached normality, meeting the criteria for normality at 3 hours. One interpretation of this result is that the process of phagocytosis is itself a randomizing system with Gaussian noise resulting from phagolysosome formation and kinetics of the initial acid-base reactions between increasing proton flux and quenching glucuronic acids in the capsular polysaccharide. With time, the titration is completed as all glucuronic acid residues are protonated. Dead cells did not synthesize additional polysaccha- phagolysosomes. On the other hand, a less acidic phagosomal $\mathrm{pH}$ is conducive to intracellular survival for $M$. tuberculosis (49). During an infectious process when the immune system confronts numerous microbial cells, the random nature of the final phagosomal $\mathrm{pH}$ will result in some fraction of the infecting inoculum being controlled and possibly killed by initial ingestion, allowing antigen presentation. In this regard, the mean number of bacteria in the phagolysosomes and cytoplasm of macrophages infected with the intracellular pathogen Francisella tularensis exhibits stochastic dynamics (50), which in turn could result from the type of stochastic processes in phagolysosome formation noted here. Hence, chance in phagolysosomal $\mathrm{pH}$ acidification provides phagocytic cells with a mechanism to hedge their bets such that the stochastic nature of the process is itself a host defense mechanism.

In biology, bet-hedging was described by Darwin as a strategy to overcome an unpredictable environment (51), which is now known as diversified bet-hedging: diversifying offspring genotype to ensure survival of at least some individuals at the expense of reducing the mean inclusive fitness of the parent. The main 
idea behind any bet-hedging strategy, under the assumption of multiplicative fitness, is that to maximize long-term fitness, an organism must lower its variance in fitness between generations (52-54). For example, varying egg size and number in a clutch can bank against years with a hostile environment in a form of diversified bet-hedging (55). During any given good year, fewer of the offspring will thrive because some are specifically designated for survival in bad years, drastically increasing fitness during bad years at the cost of a slight fitness reduction during good. Our observations suggest that, as a population, macrophages perform a bet-hedging strategy by introducing a $\mathrm{pH}$ level as inhospitable to pathogens as possible, while still maintaining biologically possible levels. However, such an approach could select for acidresistant microbes. Our observation suggests that to avoid an arms race, the macrophage not only lowers the $\mathrm{pH}$ level to a level that is unfavorable to most microbes, since the reduction in $\mathrm{pH}$ level reduces the optimal growth condition to around $70 \%$ of the optimum (see Figure 3 red line under the blue shaded curve), but it also introduces randomness in the achieved $\mathrm{pH}$, such that ingested microbes are less likely to adapt to the potentially hostile environment. In other words, tightly controlled $\mathrm{pH}$ reduction by the host without increased variation, might introduce an evolutionary arms race between pathogens and their host cells, leading toward a deleterious outcome of selecting microbial acid resistance. A similar evolutionary arms race would occur between environmental C. neoformans and the amoebas who prey on them. Previous studies indicate that acidification in amoebas closely resembles that of macrophages with similar final $\mathrm{pH}$ and time to acidification $(56,57)$. Thus, phagocytic predators in soil would face the same problem as macrophages in not knowing the $\mathrm{pH}$ tolerance of their prey. It is conceivable that they employ a similar defense strategy to that observed in macrophages, likely to have been honed in by eons of selection in soil predator-prey interactions.

Additionally, our model shows that even at the lower extreme mean $\mathrm{pH}$, macrophage populations still benefit in the long run by increasing their phagolysosomal $\mathrm{pH}$ variance. We note that the $\mathrm{pH}$ of other mammalian fluids such as that of the blood are tightly regulated such that their physiological variance is very small (58). For example, human plasma $\mathrm{pH}$ averages 7.4 with a range of only 0.05 units. Hence, organisms can maintain tight $\mathrm{pH}$ control when it is physiologically important, implying that the comparatively large range of phagolysosomal $\mathrm{pHs}$ measured in all conditions studied is a designed feature of this system. In other words, one can envision the macrophage's first line of defense of bet-hedging phagolysosomal $\mathrm{pH}$ as a roulette wheel, where the payout is likelihood of macrophage survival and the placed bet is a range of possible $\mathrm{pH}$. Placing multiple bets across the table (increasing standard deviation of $\mathrm{pH}$ distribution) increases the chance of winning at the cost of a lower payout (mean fitness), resulting in a more profitable long-term strategy (increased mean log fitness). In fact, the most profitable roulette strategy is broad color bets with lower payouts but the best winning probability. The most profitable betting strategy would of course be to play Blackjack instead, but macrophages do not have that luxury.

We observed that this bet-hedging strategy was displayed in M1 polarized macrophages, dependent on a normal distribution of phagolysosomal $\mathrm{pH}$ with a high variance. M2 macrophages, which acidify with different dynamics to M1 (59), do not acidify to a normal distribution and thus do not engage in this bet-hedging strategy. Additionally, we found that M2-skewed macrophage populations on average acidify to a higher $\mathrm{pH}$ than M1-skewed populations and are overall more favorable to C. neoformans, an observation supported by previous literature $(59,60)$. This hypothesis, while requiring more investigation, may help explain why M2-skewed macrophages are unable to control C. neoformans infection, as their phagolysosomes acidify to a $\mathrm{pH}$ range that is optimal for C. neoformans growth (15).

Additionally, intracellular pathogens have developed their own ways to disrupt or game the macrophage betting system. For C. neoformans, which can manipulate phagolysosomal $\mathrm{pH}$ through several mechanisms, our model shows that the fungal-mediated changes in the distribution of phagolysosomal $\mathrm{pH}$ favors the pathogen overall, disrupting the bet-hedging system and resulting in lower macrophage population fitness. This phenomenon can also be observed with $M$. avium in which previously reported phagolysosomal $\mathrm{pH}$ values (35) show a marked increase in mean log fitness when the ingested pathogen is killed and unable to modulate $\mathrm{pH}$. This finding is emphasized by our analysis of phagolysosomes whose $\mathrm{pH}$ has been pharmacologically manipulated to a region favorable to pathogens. We found that disrupting the macrophage betting system this way led to a drastically reduced overall mean $\log$ fitness of the macrophage population.

Given that acid has potent antimicrobial properties, one might wonder why phagolysosomal acidification does not reach even lower and more acidic pHs. There are several explanations for observed lower limits in $\mathrm{pH}$. Acidification is achieved by pumping protons into the vacuole, and achieving lower $\mathrm{pHs}$ against an ever-increasing acidity gradient could prove thermodynamically difficult. There is also evidence that the integrity of the cell membrane lipid bilayer is compromised by acidity at a $\mathrm{pH}$ of 3 and below $(61,62)$, which could promote leakage of phagolysosomal contents into the cytoplasm with damage to the host cell. Consequently, we propose that a larger variation in macrophage phagolysosomal $\mathrm{pH}$ acts as a diversified bet-hedging strategy against the stochasticity of the potential $\mathrm{pH}$ tolerances of ingested microbes within the physiological limits of achievable acidification. This hypothesis is supported by simulated data in which an increase in standard deviation of the $\mathrm{pH}$ distribution slightly lowers the expected mean but significantly decreases the standard deviation of host survival. Fully analyzing the consequences and evolutionary tradeoff of this strategy would require a closer analysis that considers the costs and benefits with regard to the host. Though outside the scope of our current work, our observations suggest this line of investigation for future studies.

Our results delineate new avenues for investigation. Most perplexing is how a random phagolysosomal $\mathrm{pH}$ is established and maintained. One can imagine various mechanisms including variation in the number of ATPase molecules and/or differences in activity of individual pumps depending on location and adjoining structures. To resolve this would require sophisticated technology allowing the measurement of $\mathrm{pH}$ in individual phagolysosomes as a function of pump occupancy and efficacy. Such techniques are likely beyond the current technological horizon but suggest new fertile areas of scientific investigation. From a clinical 
perspective, a drug that increases variation in phagolysosomal $\mathrm{pH}$ could be useful in enhancing macrophage antimicrobial efficacy in situations where one cannot anticipate which specific microbes will be encountered. On the other hand, drugs that specifically modulate $\mathrm{pH}$ may be useful in situations with specific microbes that express marked acid/base tolerance. In this regard, chloroquine alkalizes $C$. neoformans phagolysosomes, moving away from optimal inhibitory $\mathrm{pH}$. This drug has been shown to enhance macrophage activity against $C$. neoformans $(9,63)$, an outcome predicted by our model.

In summary, we document that phagolysosomal acidification, a critical process for phagocytic cell efficacy in controlling ingested microbial cells, manifests stochastic dynamics that permit a bet-hedging strategy for phagocytic cells ingesting microbes of unknown $\mathrm{pH}$ tolerance. These observations establish that the use of bet-hedging strategies in biology extends to the suborganism level to involve cells and their organelles. This, in turn, implies a significant role for chance in the resolution of conflict between microbes and host phagocytic cells in individual phagolysosomes. We have recently argued that chance is also a major determinant of individual susceptibility to infectious diseases at the organismal level (64). Variability in the outcome of infectious disease among individual hosts may reflect the sum of innumerable chance events for host-microbe interactions at the cellular level, which include the process of phagosome acidification.

\section{Methods}

Cell strains and culture conditions. C. gattii species complex strains R265, WM179, and WM161 were obtained from ATCC, and C. neoformans species complex serotype A strain H99 as well as ure14 (lacking urease, derived from H99) were originally obtained from John Perfect (Durham, North Carolina, USA). The strains were stored at $80^{\circ} \mathrm{C}$. Frozen stocks were streaked onto Sabouraud dextrose agar (SAB) and incubated at $30^{\circ} \mathrm{C}$. Liquid suspensions of cryptococcal cultures were grown in $\mathrm{SAB}$ overnight at $30^{\circ} \mathrm{C}$. Cryptococcal cultures were heat killed by incubating at $65^{\circ} \mathrm{C}$ for 1 hour.

Macrophage cells were either mouse BMDMs obtained from 6-week-old C57BL/6 female mice from The Jackson Laboratory, J774.16 macrophage-like cells, or human monocytes isolated from PBMCs. BMDMs were isolated from hind leg bones and for differentiation were seeded in $10-\mathrm{cm}$ tissue culture-treated dishes (Corning) in Dulbecco's modified Eagle medium (DMEM, Corning) with $10 \%$ FBS (Atlanta Biologicals), 1\% nonessential amino acids (Cellgro), 1\% penicillin-streptomycin (Corning), $2 \mathrm{mM} \mathrm{Glu-}$ tamax (Gibco), 1\% HEPES buffer (Corning), 20\% L-929 cell-conditioned supernatant, and $0.1 \% \beta$-mercaptoethanol (Gibco) for 6 days at $37^{\circ} \mathrm{C}$ and $9.5 \% \mathrm{CO}_{2}$. $\mathrm{BMDMs}$ were used for experiments within 5 days after differentiation. J774.16 cells were cultured in DMEM with 10\% FBS, 1\% nonessential amino acids, 10\% NCTC109 (Gibco), and $1 \%$ penicillin-streptomycin at $37^{\circ} \mathrm{C}$ with $9.5 \% \mathrm{CO}_{2}$. For human peripheral blood mononuclear cells (hPBMCs), CD14 ${ }^{+}$ monocytes were isolated using Dynabeads Untouched Human Monocytes Kit (Thermo Fisher Scientific) according to the manufacturer's protocol. The isolated cells were differentiated in RPMI1640 medium (RPMI) with 10\% FBS (Atlanta Biologicals), 1\% penicillin-streptomycin (Corning), and $25 \mathrm{ng} / \mathrm{mL}$ humangranulocytemacrophage colony-stimulating factor (GM-CSF, MilliporeSigma) for 5 days. Human cells were further cultured in RPMI (RPMI) with
10\% FBS (Atlanta Biologicals), 1\% penicillin-streptomycin (Corning), and activated with $0.5 \mu \mathrm{g} / \mathrm{mL}$ lipopolysaccharide (LPS, MilliporeSigma) and $10 \mathrm{ng} / \mathrm{mL} \mathrm{IFN-} \gamma$ (Roche) for M1 polarization or 20 $\mathrm{ng} / \mathrm{mL}$ IL-4 for M2 polarization.

Phagolysosomal pH measurement. Phagolysosomal $\mathrm{pH}$ was measured using ratiometric fluorescence imaging involving the use of $\mathrm{pH}$-sensitive probe Oregon Green 488 as described in prior studies (15). The $\mathrm{pH}$ values analyzed here were collected in part during prior studies of C. neoformans-macrophage interactions (14-16). Briefly, Oregon Green 488 was first conjugated to monoclonal antibody $(\mathrm{mAb}) 18 \mathrm{~B} 7$, which binds $C$. neoformans capsular polysaccharide, using an Oregon Green 488 Protein Labeling Kit (Molecular Probes). The labeling procedure was done by following the manufacturer's instruction. BMDMs were plated at a density of $1.25 \times 10^{5}$ cells/well or differentiated human macrophages were plated at a density of $2.5 \times 10^{5}$ cells/well on a 24 -well plate with $12-\mathrm{mm}$ circular coverslip. Cells were activated with $0.5 \mu \mathrm{g} / \mathrm{mL}$ LPS and $100 \mathrm{U} /$ $\mathrm{mL} \mathrm{IFN}-\gamma$ or $20 \mathrm{ng} / \mathrm{mL} \mathrm{IL}-4$ as previously described at $37^{\circ} \mathrm{C}$ in a $9.5 \%$ $\mathrm{CO}_{2}$ (BMDMs) or $5 \% \mathrm{CO}_{2}$ (human macrophages) atmosphere overnight. Before infection, 2-day-old live, heat-killed H99, R265, WM179, ure1, cap59, or anti-mouse IgG-coated polystyrene beads $\left(3.75 \times 10^{6}\right.$ cells or beads/mL) were incubated with $10 \mu \mathrm{g} / \mathrm{mL}$ Oregon Green 488-conjugated $\mathrm{mAb} 18 \mathrm{~B} 7$ for 15 minutes. Macrophages were then incubated with Oregon Green 488-conjugated mAb 18B7-opsonized particles in $3.75 \times$ $10^{5}$ cryptococcal cells or beads per well (BMDMs) or $2.5 \times 10^{5} \mathrm{cell} /$ well (human macrophages). For drug treatment experiments, the macrophage cell media were supplemented with $3 \mu \mathrm{M}$ chloroquine. Cells were either centrifuged immediately at $350 \mathrm{~g}$ for 1 minute or incubated at $4^{\circ} \mathrm{C}$ for 30 minutes to synchronize ingestion and cultures were incubated at $37^{\circ} \mathrm{C}$ for 10 minutes to allow phagocytosis. Extracellular cryptococcal cells or beads were removed by washing 3 times with fresh medium, a step that prevents the occurrence of new phagocytic events. As an additional safeguard against new phagocytic events, fresh media was supplemented with Alexa Fluor 568-conjugated mAb $18 \mathrm{~B} 7$ for 1 hour to label extracellular particles. Samples on coverslips were collected at their respective time points after phagocytosis by washing twice with prewarmed HBSS and placing upside down on MatTek Petri dish (MatTek) with HBSS in the microwell. Images were taken by using Olympus AX70 microscopy with a $\times 40$ objective at dual excitation $440 \mathrm{~nm}$ and $488 \mathrm{~nm}$, and emission $520 \mathrm{~nm}$. Images were analyzed using MetaFluor Fluorescence Ratio Imaging Software (Molecular Devices). Fluorescence intensities were used to determine the ratios of Ex $488 \mathrm{~nm} / \mathrm{Ex} 440$ $\mathrm{nm}$ that were converted to absolute $\mathrm{pH}$ values using a standard curve where the images are taken as above but intracellular $\mathrm{pH}$ of macrophages was equilibrated by adding $10 \mu \mathrm{M}$ nigericin in $\mathrm{pH}$ buffer $(140 \mathrm{mM} \mathrm{KCl}$, $1 \mathrm{mM} \mathrm{MgCl}, 1 \mathrm{mM} \mathrm{CaCl}_{2}, 5 \mathrm{mM}$ glucose, and appropriate buffer $\mathrm{pH} 5.0$ or lower: acetate-acetic acid; $\mathrm{pH} 5.5$ to 6.5: MES; pH 7.0 or more: HEPES. Desired $\mathrm{pH}$ values were adjusted using either $1 \mathrm{M} \mathrm{KOH}$ or $1 \mathrm{M} \mathrm{HCl})$. The $\mathrm{pH}$ of buffers was adjusted at 3 to 7 using $0.5 \mathrm{pH}$ unit increments.

Immunofluorescence microscopy. BMDMs were seeded on 12-mm circular coverslips in 24-well tissue culture plates at $2.5 \times 10^{5}$ cells per well. Cells were activated with $0.5 \mu \mathrm{g} / \mathrm{mL}$ LPS and $100 \mathrm{U} / \mathrm{mL}$ IFN- $\gamma$ at $37^{\circ} \mathrm{C}$ in a $9.5 \% \mathrm{CO}_{2}$ atmosphere overnight. Anti-mouse IgG-coated beads were opsonized with $10 \mu \mathrm{g} / \mathrm{mL}$ and added to cells at MOI 1 . Phagocytosis was synchronized by centrifuging the plate at $350 g$ for 1 minute before being incubated at $37^{\circ} \mathrm{C}$. At each time point, media was replaced with $4 \%$ paraformaldehyde (PFA) and incubated for 10 minutes at room temperature to fix cells. PFA was removed and cells were 
washed 3 times with $1 \mathrm{~mL}$ PBS (1X). Coverslips were blocked for 1 hour at room temperature with $2 \%$ BSA in PBS. Primary incubation was performed with Rb $\alpha E E A 1$ (Thermo Fisher Scientific, MA5-14794) at 1:100 dilution or Rb $\alpha$ V-ATPase (Thermo Fisher Scientific, PA5-29899) at 1:100 dilution in blocking buffer for 1 hour at room temperature. Coverslips were washed with blocking buffer before secondary incubation with goat $\alpha$ Rb Alexa Fluor 488 (1:100) in blocking buffer for 1 hour at room temperature. Coverslips were washed once more with $\mathrm{dH}_{2} \mathrm{O}$ and mounted on glass slides using Prolong Gold mounting agent. Slides were imaged via a Zeiss Axiovert 200M microscope and intensity was analyzed using Zeiss Lite Blue Version. A region of interest was generated by outlining the ingested bead via phase contrast channel then measuring mean fluorescence intensity of the secondary antibody.

Trained macrophage experiments. BMDMs were plated at a density of $2 \times 10^{5}$ cells/well on 24 -well plate with $12-\mathrm{mm}$ circular coverslip. Cells were activated with $0.5 \mu \mathrm{g} / \mathrm{mL}$ LPS and $100 \mathrm{U} / \mathrm{mL}$ IFN- $\gamma$; Roche and incubated at $37^{\circ} \mathrm{C}$ in a $9.5 \% \mathrm{CO}_{2}$ atmosphere overnight. Before the infection, H99 cells were stained with $0.01 \%$ Uvitex 2B for 10 minutes. BMDMs were then infected with $\mathrm{H} 99$ cells $\left(2 \times 10^{5}\right.$ cells/well $)$ in the presence of $10 \mu \mathrm{g} / \mathrm{mL}$ 18B7. After 1 hour of infection, $5 \mu \mathrm{g} / \mathrm{mL}$ amphotericin B was added to each well and the cultures were incubated for overnight. On the following day, the cultures were washed 3 times with PBS and incubated in PBS for 2 hours. After the incubation, the cultures were further washed 3 times with PBS. A fresh overnight culture of $\mathrm{H} 99$ cells was incubated with $10 \mu \mathrm{g} / \mathrm{mL}$ Oregon Green 488-conjugated mAb $18 \mathrm{~B} 7$ for 15 minutes. Macrophages were then incubated with Oregon Green 488-conjugated $\mathrm{mAb}$ 18B7-opsonized H99 cells in $2 \times 10^{5}$ cells per well. Cells were centrifuged immediately at $350 \mathrm{~g}$ for 1 minute to synchronize ingestion. Phagolysosomal $\mathrm{pH}$ were then measured using Olympus AX70 microscopy.

Time-lapse imaging and intracellular replication. The time of intracellular replication was collected by time-lapse imaging during prior studies of C. neoformans-macrophage interactions (15). For imaging, BMDMs $\left(5 \times 10^{4}\right.$ cells/well $)$ were plated on poly-D-lysine-coated coverslip bottom MatTek Petri dishes with 14-mm microwell (MatTek). Cells were cultured in completed DMEM and stimulated with $0.5 \mu \mathrm{g} / \mathrm{mL}$ LPS and $100 \mathrm{U} / \mathrm{mL}$ IFN- $\gamma$ overnight at $37^{\circ} \mathrm{C}$ with $9.5 \% \mathrm{CO}_{2}$. On the following day, macrophages were infected with cryptococcal cells (H99 or ure1;1.5 $\times 10^{5}$ cells/well) opsonized with $18 \mathrm{~B} 7(10 \mu \mathrm{g} / \mathrm{mL})$. After 2 hours of incubation to allow phagocytosis, extracellular cryptococcal cells were removed by washing the culture 5 times with fresh medium. Images were taken every 4 minutes for 24 hours using a Zeiss Axiovert 200M inverted microscope with a $\times 10$ phase objective in an enclosed chamber at $9.5 \% \mathrm{CO}_{2}$ and $37^{\circ} \mathrm{C}$. The time intervals to initial replication of individual cryptococcal cells inside macrophages were measured by time-lapse imaging.

Data processing. Phagolysosome $\mathrm{pH}$ intervals were calculated by subtracting measured $\mathrm{pH}$ levels of phagolysosomes from a starting $\mathrm{pH}$ of 7.2 and the $\mathrm{pH}$ of the surrounding media, and individual interval measurements were concatenated into a single data set for each time point examined.

Data analysis. Discrimination of deterministic versus stochastic dynamics was achieved using the previously characterized permutation spectrum test (21). In this method, the processed data sets were segmented into overlapping subsets of 4 data points using a sliding window approach, as detailed in Figure 4, and assigned 1 of 24 (4!) possible ordinal patterns based on the ordering of the 4 terms in the subset. The frequencies with which each unique ordinal pattern occurred in the data set were then calculated and plotted. Determin- istic dynamics were characterized by the occurrence of forbidden ordinals, equal to ordinal patterns that don't occur in the data set, whereas stochastic dynamics were characterized by the presence of all ordinals. Measured phagolysosomal pHs were subtracted from an initial $\mathrm{pH}$ value (7.2) based on cell media $\mathrm{pH}$ and placed in a vector. Subsets of 4 data points were generated using a sliding window approach in which the first 4 values were grouped, the window shifted by 1 , and the subsequent set of 4 values grouped. Each subset was prescribed an ordinal pattern based on the relative values of the data points in the subset to each other with, for instance, the lowest value assigned a $\mathrm{O}$ in the ordinal pattern and the highest assigned a 3. Further characterization of deterministic dynamics was achieved using the previously characterized point count plot (65), in which periodic versus chaotic dynamics were differentiated based on the distribution of peaks in the calculated power spectrum of each data set. Power spectrums were estimated with Matlab's Lomb-Scargle power spectral density (PSD) estimate function and subsequently normalized. From the normalized power spectrum, point count plots were generated by counting the number of peaks above a set threshold - the point threshold - with values of the point threshold ranging from 0 to 1 . Periodic dynamics were characterized by staircase point count plots whereas chaotic dynamics were characterized by point count plots with a decreasing exponential shape.

Distribution and normality analysis. Each set of sample data was fit to a series of distributions using the R package fitdistrplus with default parameters for each distribution type, generating the histograms and Q-Q plots. Normality and significance were calculated via the base $\mathrm{R}$ Shapiro-Wilk test.

Generating and analyzing simulations. Let $\mathrm{f}(\mathrm{x})$ be the probability distribution of the host's $\mathrm{pH}$. For simplicity, we assume that $\mathrm{f}$ is normal $(\mu, \sigma)$. Let $\mathrm{F}(\mathrm{x})$ be the corresponding cdf. At every time point, we randomly sample a pathogen $\mathrm{i}$ from a set of pathogens. The pathogen has a range $\left(a_{i}, b_{i}\right)$ of viable $\mathrm{pH}$, which differs among pathogens. Minimum tolerable $\mathrm{pH}$ values were obtained from the literature as described, while a constant value of 8 was used for maximum since most pathogens were inhibited as high $\mathrm{pH}$ as well as phagolysosomes being unlikely to alkalize. The host, in turn, randomly chooses a $\mathrm{pH}$ by sampling from the distribution $\mathrm{f}(\mathrm{x})$. If the host's $\mathrm{pH} \mathrm{x}$ falls in the interval $\left(a_{i}, b_{i}\right)$, then there is a probability p of the host dying. We can think of the host's survival rate $\rho$ as its fitness, which is a random variable that depends on the randomness in pathogens. As a function of $\mathrm{i}$, we have $\rho(\mathrm{i})=\mathrm{p}^{*}\left(\mathrm{~F}\left(b_{i}\right)-\mathrm{F}\left(a_{i}\right)\right)$; the random variable $\rho$ assumes the values $\rho$ (i) with equal probability. Additionally, since biologically relevant phagolysosome $\mathrm{pH}$ values will only fall within a certain range, we decided to limit the possible $\mathrm{pH}$ values between 2 and $7.2 \mathrm{pH}$ of associated cell media). Any values generated outside those limits were instead recorded as their respective limit. Thus, distributions become less normally distributed at the extremes, sacrificing normality for biological practicality, as real phagolysosomes would not alkalize or be likely to acidify below 2 . In these situations, we calculated outputs based on 1000 replicates of 10,000 simulated phagolysosomes each.

Statistics. A variety of tests were used throughout this manuscript. All tests used an a priori $P$ cutoff of 0.05 to determine significance. Comparing multiple phagolysosomes within a single cell used a 2-tailed Student's $t$ test to determine differences between the initial populations. To test the normality of distributions, we used the Shapiro-Wilk normality test. To determine linear correlation between 
fluorescence intensity and HPI, we employed a linear regression according to the model intensity as a function of hours postinfection. To investigate the differences in $\mathrm{pH}$ between trained and untrained macrophages we used the 2-tailed Student's $t$ test.

Study approval. The present studies in animal and/or human cell lines were reviewed and approved by an appropriate institutional review board. Murine macrophages were harvested from mice under protocols approved by the Animal Care and Use Committee for Johns Hopkins University (Baltimore, Maryland, USA). Human peripheral blood monocytes were a gift from the laboratory of Andrea Cox (Department of Medicine, Johns Hopkins University School of Medicine Baltimore, Maryland, USA) from deidentified human leuko packs obtained from the Anne Arundel Medical Blood Center (Anne Arundel, Maryland, USA) in protocols approved by the Johns Hopkins IRB.

\section{Author contributions}

QD, KMS, AB, and AC conceived the study. QD, KMS, YS, MSF, $\mathrm{CY}, \mathrm{AB}$, and $\mathrm{AC}$ developed the methodology. QD, KMS, YS, and
AV were responsible for software. QD carried out formal analysis. QD, KMS, YS, MSF, CY, OIY, CMDLR, JBF, AB, and AC conducted investigations. $\mathrm{QD}, \mathrm{KMS}, \mathrm{MSF}, \mathrm{AB}$, and $\mathrm{AC}$ wrote the original draft. QD, KMS, YS, MSF, CY, OIY, CMDLR, JBF, AB, and $\mathrm{AC}$ reviewed and edited the manuscript. $\mathrm{QD}$ was responsible for visualization.

\section{Acknowledgments}

Arturo Casadevall was supported by grants R01HL059842, 5R01AI033774, 5R37AI033142, and 5R01AI052733. Quigly Dragotakes is supported by a fellowship from the Achievement Rewards for College Scientists (ARCS) Foundation Metro-Washington Chapter as well as grant NIH T32.

Address correspondence to: Arturo Casadevall, Johns Hopkins School of Public Health, 615 North Wolfe Street, Room E5132, Baltimore, Maryland 21205. Phone: 410.955.3457; Email: acasadevall@jhu.edu.
1. Broderick NA. A common origin for immunity and digestion. Front Immunol. 2015;6:72.

2. Rosales C, Uribe-Querol E. Phagocytosis: a fundamental process in immunity. Biomed Res Int. 2017;2017:9042851.

3. Sun-Wada GH, Tabata H, Kawamura N, Aoyama M, Wada Y. Direct recruitment of $\mathrm{H}+$-ATPase from lysosomes for phagosomal acidification. JCell Sci. 2009;122(Pt 14):2504-2513.

4. Kinchen JM, Ravichandran KS. Phagosome maturation: going through the acid test. Nat Rev Mol Cell Biol. 2008;9(10):781-795.

5. Jankowski A, Scott CC, Grinstein S. Determinants of the phagosomal pH in neutrophils. J Biol Chem. 2002;277(8):6059-6066.

6. Bouvier G, Benoliel AM, Foa C, Bongrand P. Relationship between phagosome acidification, phagosome-lysosome fusion, and mechanism of particle ingestion. JLeukoc Biol. 1994;55(6):729-734.

7. Geisow MJ, D'Arcy Hart P, Young MR. Temporal changes of lysosome and phagosome $\mathrm{pH}$ during phagolysosome formation in macrophages: studies by fluorescence spectroscopy. J Cell Biol. 1981;89(3):645-652.

8. Feldmesser M, Kress Y, Novikoff P, Casadevall A. Cryptococcus neoformans is a facultative intracellular pathogen in murine pulmonary infection. Infect Immun . 2000;68(7):4225-4237.

9. Levitz SM, Nong SH, Seetoo KF, Harrison TS, Speizer RA, Simons ER. Cryptococcus neoformans resides in an acidic phagolysosome of human macrophages. Infect Immun. 1999;67(2):885-890.

10. Ma H, Croudace JE, Lammas DA, May RC. Expulsion of live pathogenic yeast by macrophages. Curr Biol. 2006;16(21):2156-2160.

11. Alvarez M, Casadevall A. Phagosome extrusion and host-cell survival after Cryptococcus neoformans phagocytosis by macrophages. Curr Biol. 2006;16(21):2161-2165.

12. Ma H, Croudace JE, Lammas DA, May RC. Direct cell-to-cell spread of a pathogenic yeast. BMC Immunol. 2007;8:15.

13. Alvarez M, Casadevall A. Cell-to-cell spread and massive vacuole formation after Cryptococcus neoformans infection of murine macrophages. BMC Immunol. 2007;8:16.

14. De Leon-Rodriguez CM, et al. The outcome of the Cryptococcus neoformans-macrophage interaction depends on phagolysosomal membrane integrity. JImmunol. 2018;201(2):583-603.

15. Fu MS, et al. Cryptococcus neoformans urease affects the outcome of intracellular pathogenesis by modulating phagolysosomal pH. PLoS Pathog. 2018;14(6):e1007144

16. Freij JB, et al. Conservation of intracellular pathogenic strategy among distantly related Cryptococcal species. Infect Immun . 2018;86(7):e00946-17.

17. De Leon-Rodriguez CM, Fu MS, Çorbali MO, Cordero RJB, Casadevall A. The capsule of Cryptococcus neoformans modulates phagosomal pH through its acid-base properties. mSphere. 2018;3(5):e00437-18.

18. DeLeon-Rodriguez CM, Casadevall A. Cryptococcus neoformans: tripping on acid in the phagolysosome. Front Microbiol. 2016;7:164.

19. Smith LM, Dixon EF, May RC. The fungal pathogen Cryptococcus neoformans manipulates macrophage phagosome maturation. Cell Microbiol. 2015;17(5):702-713.

20. Davis MJ, et al. Cryptococcus neoformansinduced macrophage lysosome damage crucially contributes to fungal virulence. J Immunol. 2015;194(5):2219-2231.

21. Zunino L, Soriano MC, Rosso OA. Distinguishing chaotic and stochastic dynamics from time series by using a multiscale symbolic approach. Phys Rev E Stat Nonlin Soft Matter Phys. 2012;86(4 Pt 2):046210.

22. McCullough M, Sakellariou K, Stemler T, Small M. Counting forbidden patterns in irregularly sampled time series. I. The effects of under-sampling, random depletion, and timing jitter. Chaos. 2016;26(12):123103.

23. Sakellariou K, McCullough M, Stemler T, Small M. Counting forbidden patterns in irregularly sampled time series. II. Reliability in the presence of highly irregular sampling. Chaos. 2016;26(12):123104.

24. Rosso OA et al. Noisy-chaotic time series and the forbidden/missing patterns paradigm [preprint] https://arxiv.org/abs/1110.0776. Posted on aRxiv October 4, 2011.

25. Skiadas CH, Skiadas C. Handbook of Applications of Chaos Theory. Boca Raton, Florida, USA: Taylor \& Francis: 2016.

26. Fierer N, Jackson RB. The diversity and biogeography of soil bacterial communities. Proc Natl Acad Sci USA. 2006;103(3):626-631.

27. Baath K, Arnebrant K. Growth rate and response of bacterial communities to $\mathrm{pH}$ in limed and ash treated forest soils. Soil Biol Biochem. 1994;26(8):995-1001.

28. Bååth E, Frostegård A, Fritze H. Soil Bacterial biomass, activity, phospholipid fatty acid pattern, and $\mathrm{pH}$ tolerance in an area polluted with alkaline dust deposition. Appl Environ Microbiol. 1992;58(12):4026-4031.

29. Karlin S, Lieberman U. Random temporal variation in selection intensities: case of large population size. Theor Popul Biol. 1974;6(3):355-382.

30. Quintin J, et al. Candida albicans infection affords protection against reinfection via functional reprogramming of monocytes. Cell Host Microbe. 2012;12(2):223-232.

31. Sau K, Mambula SS, Latz E, Henneke P, Golenbock DT, Levitz SM. The antifungal drug amphotericin B promotes inflammatory cytokine release by a Toll-like receptor- and CD14-dependent mechanism. J Biol Chem. 2003;278(39):37561-37568.

32. Casadevall A, Coelho C, Alanio A. Mechanisms of Cryptococcus neoformans-mediated host damage. Front Immunol. 2018;9:855.

33. Coelho C, et al. Macrophage mitochondrial and stress response to ingestion of Cryptococcus neoformans. JImmunol. 2015;194(5):2345-2357.

34. Alspaugh JA, Granger DL. Inhibition of Cryptococcus neoformans replication by nitrogen oxides supports the role of these molecules as effectors of macrophage-mediated cytostasis. Infect Immun. 1991;59(7):2291-2296. 
35. Oh YK, Straubinger RM. Intracellular fate of Mycobacterium avium: use of dual-label spectrofluorometry to investigate the influence of bacterial viability and opsonization on phagosomal $\mathrm{pH}$ and phagosome-lysosome interaction. Infect Immun. 1996;64(1):319-325.

36. Griffiths $\mathrm{G}$. On phagosome individuality and membrane signalling networks. Trends Cell Biol. 2004;14(7):343-351.

37. Mallik KC, Banerjee PL, Chatterjee BD, Pramanick $M$. An experimental study of the course of infection in mice after intranasal insufflation with Cryptococcus neoformans. Indian JMed Res. 1966;54(7):608-610.

38. Paul D, Achouri S, Yoon YZ, Herre J, Bryant CE, Cicuta P. Phagocytosis dynamics depends on target shape. Biophys J. 2013;105(5):1143-1150.

39. Dragotakes Q, Fu MS, Casadevall A. Dragotcytosis: elucidation of the mechanism for Cryptococcus neoformans macrophage-to-macrophage transfer. Jimmunol. 2019;202(9):2661-2670.

40. Cherniak R, Valafar H, Morris LC, Valafar F. Cryptococcus neoformans chemotyping by quantitative analysis of $1 \mathrm{H}$ nuclear magnetic resonance spectra of glucuronoxylomannans with a computer-simulated artificial neural network. Clin Diagn Lab Immunol. 1998;5(2):146-159.

41. Westman J, Moran G, Mogavero S, Hube B, Grinstein S. Candida albicans hyphal expansion causes phagosomal membrane damage and luminal alkalinization. mBio. 2018;9(5):e01226-18.

42. Weber SM, Levitz SM. Chloroquine antagonizes the proinflammatory cytokine response to opportunistic fungi by alkalizing the fungal phagolysosome. J Infect Dis. 2001;183(6):935-942.

43. Harrison TS, Griffin GE, Levitz SM. Conditional lethality of the diprotic weak bases chloroquine and quinacrine against Cryptococcus neofor- mans. J Infect Dis. 2000;182(1):283-289.

44. Artavanis-Tsakonas K, Love JC, Ploegh HL, Vyas JM. Recruitment of CD63 to Cryptococcus neoformans phagosomes requires acidification. Proc Natl Acad Sci USA. 2006;103(43):15945-15950.

45. Mantegazza AR, et al. NADPH oxidase controls phagosomal $\mathrm{pH}$ and antigen cross-presentation in human dendritic cells. Blood. 2008;112(12):4712-4722.

46. Savina A, et al. NOX2 controls phagosomal pH to regulate antigen processing during crosspresentation by dendritic cells. Cell. 2006;126(1):205-218.

47. Yates RM, Russell DG. Phagosome maturation proceeds independently of stimulation of toll-like receptors 2 and 4. Immunity. 2005;23(4):409-417.

48. Rathman M, Sjaastad MD, Falkow S. Acidification of phagosomes containing Salmonella typhimurium in murine macrophages. Infect Immun. 1996;64(7):2765-2773.

49. Queval CJ, Brosch R, Simeone R. The macrophage: a disputed fortress in the battle against Mycobacterium tuberculosis. Front Microbiol. 2017;8:2284.

50. Gillard JJ, Laws TR, Lythe G, Molina-París C. Modeling early events in Francisella tularensis pathogenesis. Front Cell Infect Microbiol. 2014;4:169.

51. Darwin C. The Foundation of the Origin of Species: Two Essays Written in 1842 and 1844. Cambridge, United Kingdom: Cambridge University Press; 1909:

52. Slatkin M. Cascading speciation. Nature. 1974;252(5485):701-702.

53. Philippi T, Seger J. Hedging one's evolutionary bets, revisited. Trends Ecol Evol (Amst). 1989;4(2):41-44.

54. Bergman A, Tennenholtz M. On the natural selection of market choice. Autonomous Agents and Multi-Agent Systems. 2002;5(4):387-395.
55. Olofsson H, Ripa J, Jonzén N. Bet-hedging as an evolutionary game: the trade-off between egg size and number. Proc Biol Sci. 2009;276(1669):2963-2969.

56. Heiple JM, Taylor DL. pH changes in pinosomes and phagosomes in the ameba, Chaos carolinensis. JCell Biol. 1982;94(1):143-149.

57. Watts C, Marsh M. Endocytosis: what goes in and how? J Cell Sci. 1992;103(Pt 1):1-8.

58. Curran RE, Claxton CR, Hutchison L, Harradine PJ, Martin IJ, Littlewood P. Control and measurement of plasma $\mathrm{pH}$ in equilibrium dialysis: influence on drug plasma protein binding. Drug Metab Dispos. 2011;39(3):551-557.

59. Davis MJ, et al. Macrophage M1/M2 polarization dynamically adapts to changes in cytokine microenvironments in Cryptococcus neoformans infection. mBio. 2013;4(3):e00264-e00213.

60. Leopold Wager CM, Wormley FL. Classical versus alternative macrophage activation: the Ying and the Yang in host defense against pulmonary fungal infections. Mucosal Immunol. 2014;7(5):1023-1035.

61. Leung CY, et al. Crystalline polymorphism induced by charge regulation in ionic membranes. Proc Natl Acad Sci USA. 2013;110(41):16309-16314.

62. Jacobsohn MK, Lehman MM, Jacobsohn GM. Cell membranes and multilamellar vesicles: influence of $\mathrm{pH}$ on solvent induced damage. Lipids. 1992;27(9):694-700.

63. Weber SM, Levitz SM, Harrison TS. Chloroquine and the fungal phagosome. Curr Opin Microbiol. 2000;3(4):349-353.

64. Casadevall A, Pirofski LA. What is a host? Attributes of individual susceptibility. Infect Immun. 2018;86(2):e00636-17.

65. Kulp CW, Zunino L. Discriminating chaotic and stochastic dynamics through the permutation spectrum test. Chaos. 2014;24(3):033116. 